\title{
A two-component model of the solar photosphere from the inversion of spectral lines
}

\author{
J. M. Borrero ${ }^{1}$ and L. R. Bellot Rubio ${ }^{2, \star}$ \\ 1 Max-Planck Institut für Aeronomie, 37191, Katlenburg-Lindau, Germany \\ 2 Instituto de Astrofísica de Canarias, 38200, Vía Láctea s/n, La Laguna, Tenerife, Spain \\ Received 29 October 2001 / Accepted 31 January 2002
}

\begin{abstract}
A two-component model of the solar photosphere is obtained from the inversion of the intensity profiles of 22 Fe I spectral lines for which very accurate atomic data (oscillator strengths, central wavelengths, and collisional broadening parameters) exist. The model is meant to describe the effects of convective motions in the solar photosphere. It has been subject to various tests to confront its predictions with observations of the solar spectrum. The model is able to reproduce the observed line shifts and equivalent widths of about 800 spectral lines of iron and other species. It is also capable of matching the observed center-to-limb variation of the continuum intensity with unprecedented accuracy. This allows us to determine line-transition parameters from the fitting of the solar spectrum. Exploratory calculations demonstrate that the model can be used to derive transition probabilities and central wavelengths of Fe I and Fe II lines, as well as other elements, within the uncertainties of the best laboratory measurements.
\end{abstract}

Key words. atomic data - line: profiles - Sun: photosphere - Sun: abundances - stars: atmospheres stars: abundances

\section{Introduction}

Determining the structure of the solar photosphere from observations is a difficult task. In the past, measurements of the continuum intensity have been used repeatedly to infer its thermal stratification. This has resulted in idealized one-component models (e.g., Holweger \& Muller 1974; Gingerich et al. 1971) capable of reproducing some features of the solar spectrum. Similar models have been obtained from the inversion of spectral lines, whereby the full shape of the observed intensity profiles is fitted in order to determine more accurate temperatures as a function of optical depth (e.g., Allende Prieto et al. 1998). The thermal properties of the solar photosphere are relatively well known by now, but unfortunately our knowledge of velocity fields is very limited. Indeed, because of their nature, one-component models are unable to describe convective motions in the photosphere of the Sun and other cool stars. This has a significant impact on our ability to model the solar spectrum. The large asymmetries exhibited by photospheric spectral lines, for example, cannot be explained in terms of one-component models even if velocity gra-

Send offprint requests to: J. M. Borrero, e-mail: borrero@linmpi.mpg.de

* Present address: Kiepenheuer-Institut für Sonnenphysik, Schöneckstr. 6, 79104, Freiburg, Germany,

e-mail: lbellot@kis.uni-freiburg.de dients are allowed, as different lines would need different gradients to be reproduced.

The availability of realistic models of the solar photosphere is important for a variety of reasons. Among them we would like to mention the determination of chemical abundances and line transition parameters. Traditionally, abundances in the solar photosphere have been obtained by fitting the equivalent widths of a number of spectral lines. For the radiative transfer, one-component models have been used in most cases (e.g., Blackwell et al. 1995a; Holweger et al. 1995). Because of their inability to produce asymmetrical profiles, these models can introduce systematic errors in the determination of equivalent widths that eventually translate into wrong abundance values. In order to minimize this source of error, multi-component model atmospheres are necessary. Another important advantage of such models is that they can be used to fit the full shape of the spectral lines, rather than their equivalent widths. This should greatly improve the accuracy of the inferred abundances because of the much greater amount of information available.

Another field where realistic models of the solar photosphere are urgently needed is the determination of atomic parameters by using the Sun as a laboratory. Accurate atomic data (transition probabilities and central wavelengths) are fundamental in many branches of astrophysics, including solar and stellar physics, interstellar 
medium and planetary sciences. However, there is a surprising paucity of reliable atomic parameters. A good example is provided by neutral iron, by far the most often used species in solar physics. About half of the Fe I lines in the wavelength range from $3000 \AA$ to $1 \mu \mathrm{m}$ have now oscillator strengths measured in the laboratory, although with varying degrees of accuracy. Many lines, especially in the ultraviolet and near infrared, are difficult to reproduce in the laboratory, so direct measurements are restricted to a small number of transitions. The situation is worse for other elements and ionization stages, for which even central wavelengths are known with limited accuracy. In many cases, one has to resort to quantum mechanical computations for estimating line transition parameters.

One way to overcome these limitations is to use the Sun as a laboratory creating the conditions (temperature, pressure and density) required by many transitions to appear. This idea has already been explored by Thévenin (1989, 1990) and Bellot Rubio et al. (1999). They determined atomic parameters by synthesizing spectral lines observed in the solar photosphere. Differences between the synthetic and observed profiles were assumed to be produced by incorrect atomic parameters, which were modified until the synthetic profiles matched the observed ones. Thévenin fitted observed equivalent widths by means of a one-component model atmosphere in order to infer oscillator strengths for a large list of spectral lines. Bellot Rubio et al. (1999) first obtained a two-component model of the solar photosphere and then derived accurate central wavelengths for a number of Fe II transitions. Since the determination of oscillator strengths and central wavelengths relies upon successful fits to observed spectral lines, it is clear that the model atmospheres employed must be as complete as possible. In particular, they should be able to reproduce the full shape of the spectral lines. This can only be achieved by using multi-component model atmospheres which take into account the velocity fields producing the observed line asymmetries. Recent improvements in 3D hydrodynamical simulations assuming LTE (Asplund et al. 2000a, 2000b) and NLTE (Shchukina \& Trujillo Bueno 2001) are able to describe the effects of convective motions in the solar photosphere to a very satisfactory degree. However, due to the extremely timeconsuming nature of these simulations, they are less appropriate than semiempirical treatments for systematic studies of the solar spectrum.

This work is devoted to obtaining a realistic twocomponent model of the solar photosphere from the inversion of spectral lines. This implies determining the stratification with depth of temperature, electron pressure and mass motions. In order to improve the degree of realism we use the most accurate atomic data available so they do not contain errors (or if they do, the least possible) that would lead to wrong stratifications of the physical parameters. Before accepting the model, it is important to show that it is able to reproduce several observational features of the solar spectrum, including the intensity contrast of granulation, convective blueshifts, and center-to-limb variation of the continuum intensity. Once these tests are carried out, the model is used to calculate atomic parameters for neutral iron and other elements. At this stage we restrict ourselves to show that a simple two-component model can be employed to determine line transition parameters with an accuracy comparable to that of the best laboratory measurements. A systematic study of the visible solar spectrum is deferred to another work. The paper is organized as follows: in Sect. 2 we describe the inversion code and details of the spectral synthesis; in Sect. 3 we present the initial guess model used for the inversion as well as the atomic data employed, and discuss the resulting model atmosphere; in Sect. 4 we perform several tests on our model and finally, we apply it to derive oscillator strengths for iron and other elements (Sect. 5).

\section{The inversion code}

The inversion code used in this work is SIR (Stokes Inversion based on Response functions; Ruiz Cobo \& del Toro Iniesta 1992). SIR iteratively modifies an initial guess model until the synthetic spectrum matches the observed one. The code can handle the full Stokes vector or the intensity profiles of as many spectral lines as desired. The basic assumptions are local thermodynamical equilibrium (LTE), homogeneous plane-parallel atmosphere, and hydrostatic equilibrium. As a result of the inversion, SIR provides the stratification of temperature, electron pressure, line-of-sight velocity, and microturbulence along the whole atmosphere. It also returns a macroturbulent velocity which is assumed to be height-independent. SIR is able to deal with one or two-component model atmospheres. In the later case, the stratification of the physical parameters is obtained independently for the two components and a filling factor is determined. The computational time is greatly reduced due to the implementation of the so-called response functions, which are the partial derivatives of the emergent intensity with respect to the free parameters.

The spectral synthesis module of SIR is based on an earlier code by Wittmann (1974) except that the hermitian algorithm described by Bellot Rubio et al. (1998) is used for the integration of the radiative transfer equation. The continuum absorption coefficient is evaluated for a given wavelength, temperature and electron pressure taking into account contributions from $\mathrm{H}, \mathrm{He}, \mathrm{H}^{-}, \mathrm{He}^{-}, \mathrm{H}_{2}^{-}$, $\mathrm{H}_{2}^{+}, \mathrm{C}, \mathrm{Mg}$, and $\mathrm{Na}$, as well as Rayleigh scattering by $\mathrm{H}$, $\mathrm{H}_{2}$ and $\mathrm{He}$, and Thomson scattering by free electrons (for additional details, see Wittmann 1974). After each iteration step, the electron pressures are put into hydrostatic equilibrium by using the equation of state of an ideal gas with variable mean molecular weight to take into account the partial ionization of the various atomic elements. Gas pressures are computed from temperatures and electron pressures on the assumptions of LTE and chemical equilibrium. In this process, the partial pressures of $\mathrm{H}, \mathrm{H}^{+}$, $\mathrm{H}^{-}, \mathrm{H}_{2}, \mathrm{H}_{2}^{+}$, and 83 other elements are determined following the strategy outlined by Mihalas (1967). 
So far, inversion techniques have been applied to obtain idealized model atmospheres capable of reproducing the shape of the observed spectral lines (see Ruiz Cobo 1998 for a review). In many cases, the observations lack spatial and/or temporal resolution. Hence, one cannot expect these semiempirical models to be perfect representations of any real structure of the solar photosphere. Hopefully, however, they grasp the basic physics needed to explain the observations. Here we are concerned with the modeling of the quiet solar photosphere, which implies modeling of convective motions. In our idealized representation, a simple two-component model describing granular and intergranular cells may suffice to reproduce the observed solar spectrum to a very reasonable degree of accuracy. If such a model exists, it could be used to determine chemical abundances and reliable atomic parameters from the fitting of the solar spectrum. Last, but not least, having a simple representation of convective motions in terms of a two-component atmosphere may be of practical interest for calibration of stellar spectra. Building 3D hydrodynamical models is a very time-consuming task, and confronting them with real observations is difficult because of the huge amount of information they provide. In any case, we do acknowledge the importance of detailed numerical simulations. Among other reasons, they help us understand some properties of semiempirical models derived from the inversion of spectral lines. The micro and macroturbulent parameters required to fit the observations are good examples of this interplay. As the 3D hydrodynamical simulations of convection by Asplund et al. (2001a) demonstrate, these parameters are largely the result of very poor spatial resolutions which do not allow to sample all the velocity fields present in the resolution element. This must be borne in mind when interpreting the results of inversion techniques.

At this point we note that microturbulence has traditionally provided an additional source for line broadening. Collisional broadening by neutral hydrogen atoms is known to be underestimated in spectral syntheses using the van der Waals interaction potential due to its asympthotic nature. In order to compensate the smaller broadening, the microtubulence needs to be overestimated. The introduction of the so-called damping enhancement factor in order to increase the broadening produced by collisions is meant to mitigate this problem. However, because of the ad-hoc nature of this correction factor, the microturbulence determined from observed spectral lines is expected to be wrong. Fortunately, the damping enhancement factor is no longer necessary since the important advances achieved on the quantum formulation of the collisional broadening (O'Mara 1976; Anstee \& O'Mara 1995; Barklem \& O'Mara 1997; Barklem et al. 1998), which makes a realistic estimation of the interaction potential with neutral perturber atoms.

This new treatment of the collisional broadening has been implemented in SIR. From test calculations we have found that it leads to similar broadenings than those resulting from van der Waals interaction potentials and enhancement factors of $2-3$ (for neutral iron). Since microturbulence is considered as a free parameter in the inversion process, with the implementation of the new treatment we hope that the degeneracy between microturbulence and collisional broadening can be broken down. This would allow us to obtain more reasonable values for microturbulent velocities.

\section{The two-component model}

\subsection{Initial guess model}

The inversion code requires an initial guess model to fit the observations. We have chosen the two-component model of the quiet Sun determined by Bellot Rubio et al. (1999) from the inversion of seven Fe I lines (see Fig. 1). Contrary to what is done in the present work, oscillator strengths were taken from Thévenin $(1989,1990)$ and pure van der Waals interaction potentials were assumed for the collisional broadening. This could have had a significant impact on the reliability of the atmospheric parameters inferred from the inversion. While the velocity fields of the model appear to be realistic, the degree of reliability of temperatures and microturbulence is much lower. In deep layers $\left(\log \tau_{5} \simeq 0\right.$ ), microturbulent velocities as high as $10 \mathrm{~km} \mathrm{~s}^{-1}$ were found, probably as a result of underestimated collisional broadenings. Temperatures are also somewhat suspicious due to the use of solar oscillator strengths. In this model, the temperature difference between granular and intergranular cells is about $80 \mathrm{~K}$ at continuum optical depth unity, which is a lower value than that derived from the continuum intensity contrast of the best granulation images $(\simeq 180 \mathrm{~K})$. In addition, the temperature difference in intermediate layers seems to be too large.

Because of these reasons, we modify the initial microturbulence so that it is height-independent and equal to a rather small value of $100 \mathrm{~m} \mathrm{~s}^{-1}$. If temperatures are to change during the inversion, the height of formation of the lines will vary and each line will be affected by velocity fields different from the initial ones. Therefore, as soon as we decide that the thermal structure of the guess model can be improved, we need to allow both the temperature and the velocity to change during the inversion.

\subsection{Spectral lines and observational data}

The reliability of model atmospheres determined from inversions strongly depends on the use of suitable spectral lines for which accurate atomic parameters exist. This is necessary to avoid systematic errors in temperature and velocity that would arise in order to compensate the effects of erroneous atomic parameters. Since the inversion code fits the shape of intensity profiles, the lines need to be free of blends unless they are considered in the spectral synthesis. Additionally, in order to avoid strong NLTE effects, it is desirable that they are not formed in high photospheric layers. Lines with equivalent widths smaller than, 

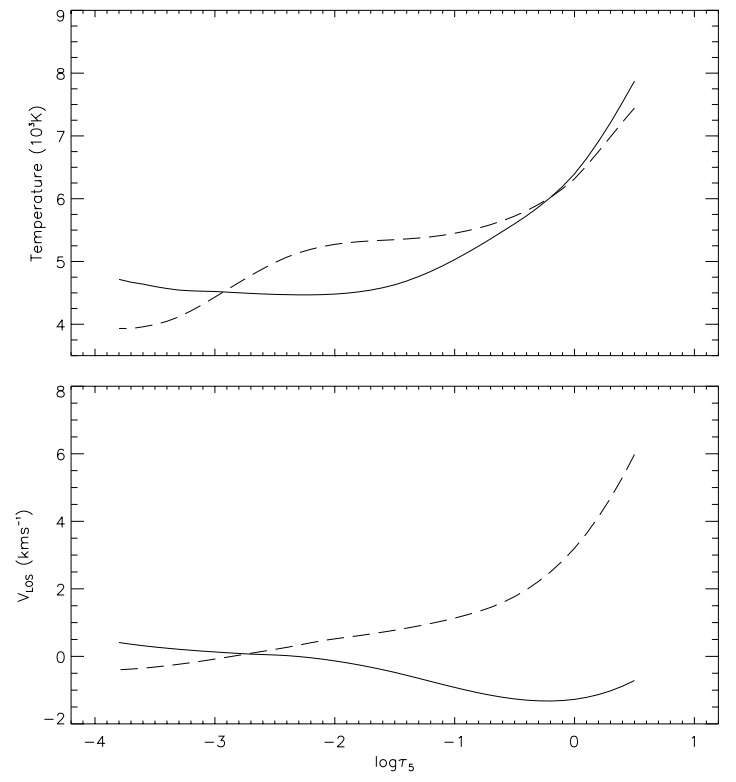

Fig. 1. Initial two-component model of the quiet sun (modification of Bellot Rubio et al. 1999). The solid and dashed lines represent the granular and intergranular flows respectively. Positive velocities indicate downflows. The gravitational redshift has been removed. The filling factor (contribution of the intergranular component) is $\alpha=0.288$. The macroturbulence is the same for granules and intergranules and equal to $0.8 \mathrm{~km} \mathrm{~s}^{-1}$. The microturbulence has been set to $0.1 \mathrm{~km} \mathrm{~s}^{-1}$ in both components.

say, $250 \mathrm{~m} \AA$ and relative core intensities greater than 0.4 are appropriate for this purpose.

Clearly, the spectral lines used in the inversion must provide enough information if we are to determine the physical parameters of the photosphere at all heights. This implies choosing lines with different excitation potentials and oscillator strengths. Since spectral lines are formed in broad height intervals, using a large number of transitions is important to ensure redundancy.

We have selected 22 Fe I lines which meet these constraints (Table 1). Iron lines dominate the solar spectrum and have been used traditionally because they provide a wide range of diagnostic capabilities. Not less important is the fact that the most accurate line transition data available are those of neutral iron. Transition probabilities listed in Table 1 have been measured in the laboratory by the Oxford (Blackwell et al. 1995a) or Hannover-Kiel (Holweger et al. 1995) groups. The central wavelengths and the excitation potentials of the lower atomic levels have been taken from Nave et al. (1994). The parameters $\alpha$ and $\sigma$ needed to calculate the collisional broadening come from Barklem et al. (2000) and Asplund et al. (2000a). We believe that these are the best atomic data available at present. The uncertainty in the laboratory measurements of central wavelengths by Nave et al. is quoted to be $\pm 3 \mathrm{~m} \AA$ at $5000 \AA$, while the uncertainties in the oscillator strengths can be estimated to be up to 0.11 dex (0.06 dex on average) according to Bard \& Kock (1994). For the spectral synthesis we adopt an iron abundance of 7.46 dex in the usual logarithmic scale. This value is appropriate for LTE analyses according to Asplund et al. (2000b).

The line profiles have been extracted from the spectral atlas of Brault \& Neckel (1987, see also Neckel 1994), which gives the spatially and temporally averaged solar intensity at disk center from 3290 to $12510 \AA$. The observations were taken with the Fourier Transform Spectrometer (FTS) attached to the McMath telescope in Kitt Peak. The signal-to-noise ratio of these observations is about 2500 with a resolving power $\Delta \lambda / \lambda \simeq 400000$. The wavelength calibration of the FTS atlas has been found to be free from systematic effects by Allende Prieto \& García López (1997). Wherever a blend is suspected in any of the lines, a mask is applied to remove the part of the profile affected by it. This leaves us with 1579 data points which sample the $22 \mathrm{Fe} I$ lines at intervals of $6 \mathrm{~mA}$.

\subsection{Results of the inversion}

Once the observed profiles, atomic data, and the initial atmosphere have been specified we must decide on the optimum number of free parameters. This is a difficult task because a compromise between different requirements is necessary. First, the code has to be given enough freedom so as to be able to produce synthetic profiles almost identical to the observed ones; this can be easily achieved by increasing the number of parameters to be determined from the inversion. On the other hand, the uniqueness of the inferred models cannot be guaranteed with too many free parameters, which could result in very unrealistic stratifications. For these reasons it is important to adopt the smallest number of parameters allowing appropriate modifications of the initial model. In the present work, the temperature and line-of-sight velocity of the two atmospheric components are perturbed by means of five parameters each. The microturbulence is modified with three more parameters for each of the two components, while the macroturbulence is assumed to be height-independent but not equal in the two components. Finally, a filling factor (fractional area of the resolution element occupied by the intergranular component) is estimated. Therefore, the total number of parameters to be determined from the inversion is 29. This value is reasonable according to our previous experience and turns out to be adequate to reproduce the observed profiles.

In order to obtain realistic atmospheres without increasing the computing time and, at the same time, keep the problem tractable, Frutiger et al. (2000) imposed the constraint of mass conservation in their inversions. While this seems reasonable, we are not sure how to constrain our velocity fields to satisfy instantaneous mass conservation, especially when one deals with spatially and temporally averaged observations. Furthermore, motions perpendicular to the line of sight are important for the total mass flux. One dimensional model atmospheres neglect these motions, making the implementation of mass conservation 
Table 1. Spectral lines used in the inversion. $\lambda_{0}$ indicates the laboratory central wavelength, $E_{\text {lower }}$ the excitation potential of the lower level, and log gf the logarithm of the oscillator strength times the multiplicity of the lower level. $\alpha$ and $\sigma$ stand for the temperature parameter and cross section for collisional broadening ( $a_{0}$ is Bohr radius). These data have been taken from: $a-$ Blackwell et al. (1995a); $b$ - Holweger et al. (1995); $c$ - Nave et al. (1994); and $d$ - Barklem et al. (1997, 1998, 2000).

\begin{tabular}{lcccc}
\hline \hline$\lambda_{0}^{c}(\dot{A})$ & $E_{\text {lower }}^{c}(\mathrm{eV})$ & $\log g f$ & $\alpha^{d}$ & $\left(\sigma / a_{0}^{2}\right)^{d}$ \\
\hline Fe I 5956.6943 & 0.859 & $-4.605^{a}$ & 0.252 & 227 \\
Fe I 6082.7104 & 2.223 & $-3.573^{a}$ & 0.271 & 306 \\
Fe I 6151.6182 & 2.176 & $-3.299^{a}$ & 0.263 & 277 \\
Fe I 6173.3354 & 2.223 & $-2.880^{a}$ & 0.266 & 281 \\
Fe I 6200.3130 & 2.608 & $-2.437^{a}$ & 0.235 & 350 \\
Fe I 6280.6182 & 0.859 & $-4.387^{a}$ & 0.253 & 223 \\
Fe I 6481.8701 & 2.279 & $-2.984^{a}$ & 0.243 & 308 \\
Fe I 6498.9390 & 0.958 & $-4.699^{a}$ & 0.253 & 226 \\
Fe I 6574.2285 & 0.990 & $-5.004^{a}$ & 0.250 & 226 \\
Fe I 6625.0220 & 1.011 & $-5.336^{a}$ & 0.253 & 226 \\
Fe I 6750.1523 & 2.424 & $-2.621^{a}$ & 0.241 & 335 \\
Fe I 6945.2051 & 2.424 & $-2.482^{a}$ & 0.243 & 331 \\
Fe I 5412.7856 & 4.434 & $-1.716^{b}$ & 0.280 & 971 \\
Fe I 5661.3457 & 4.284 & $-1.756^{b}$ & 0.229 & 735 \\
Fe I 5778.4531 & 2.588 & $-3.440^{b}$ & 0.237 & 361 \\
Fe I 5784.6582 & 3.396 & $-2.530^{b}$ & 0.244 & 796 \\
Fe I 5855.0767 & 4.607 & $-1.478^{b}$ & 0.279 & 962 \\
Fe I 6240.6460 & 2.223 & $-3.230^{b}$ & 0.272 & 301 \\
Fe I 6581.2100 & 1.458 & $-4.680^{b}$ & 0.245 & 254 \\
Fe I 6739.5220 & 1.557 & $-4.790^{b}$ & 0.244 & 256 \\
Fe I 6804.2715 & 4.584 & $-1.813^{b}$ & 0.258 & 273 \\
Fe I 6837.0059 & 4.593 & $-1.687^{b}$ & 0.258 & 273 \\
\hline & & & &
\end{tabular}

difficult. Therefore, we have decided not to impose such a constraint. Instead, we increase the amount of information by fitting 22 spectral lines, whereas Frutiger et al. used only 14 lines in their inversions.

In Fig. 2 we plot an example of observed and bestfit profiles resulting from the inversion. The equivalent signal-to-noise ratio of the fit (defined as the inverse of the standard deviation of the residuals) for the $22 \mathrm{Fe}$ I lines is 211 , with maximum relative errors of about $3 \%$. This is indeed an excellent result. The asymmetrical shape of the profiles, which is known to be the result of the velocity fields of granular and intergranular cells, is reproduced to a reasonable degree of accuracy. As already mentioned, such agreement is due to our using a two-component model atmosphere.

The model determined from the inversion (Fig. 3 and Appendix A) contains all the basic properties of granular and intergranular cells, although it cannot be considered as an exact representation of them. The granular component is found to be hotter than the intergranular component in deep layers, but cooler in intermediate layers. The crossing point occurs at $\log \tau_{5} \simeq-0.6$, while
Ruiz Cobo et al. (1996) inferred a similar temperature reversal at $\log \tau_{5} \simeq-1.4$. We find a second crossing point at $\log \tau_{5} \simeq-1.7$, where the granular component becomes hotter than the intergranular component. Given the error bars, we are not sure that this is a real feature of the solar photosphere. Indeed, the two temperature stratifications are indistinguishable within the uncertainties of the model from $\log \tau_{5} \simeq-2.8$ upwards. At $\log \tau_{5}=0$, i.e., in continuum forming layers, the temperature difference between the two components is $229 \mathrm{~K}$, which is a more realistic value compared to the $80 \mathrm{~K}$ difference of the initial model.

The velocity stratifications are found to be quite smooth, with downflows of $3.7 \mathrm{~km} \mathrm{~s}^{-1}$ in the intergranular component and upflows of $1.1 \mathrm{~km} \mathrm{~s}^{-1}$ in the granular component at $\log \tau_{5}=0$ (already corrected for gravitational redshift). We note that the larger velocities but smaller filling factor (0.24) of the intergranular flow at $\log \tau_{5}=0$ are consistent with mass conservation, although this constraint was not imposed explicitly. According to our results, the overshooting convection zone finishes in the middle photosphere $\left(\log \tau_{5} \simeq-1.8\right)$, where the line-of-sight velocity is almost identical in both components and close to $0 \mathrm{~m} \mathrm{~s}^{-1}$. The apparently weird behavior of the granular velocity field, with changes of sign along the photosphere, cannot be taken as a real feature of granular cells. In our idealized representation, the inferred granular velocities are those needed to reproduce the observations together with the intergranular velocities. The relatively large upflows of the intergranular component in high layers should compensate to some extent with the small downflows of the granular component, so that in this region the velocity fields of the model are compatible with nearly zero granular and intergranular velocities (what we expect for the real Sun).

Another important result concerns the microturbulence derived from the inversion: while in granules it seems to be more or less the same before and after the inversion, the microturbulence for intergranules grows up to $2.05 \mathrm{~km} \mathrm{~s}^{-1}$. This is in agreement with the results of Frutiger et al. (2000) and Nesis et al. (1992), with the only difference that we do not find the microturbulence to vary along the photosphere.

In Fig. 3 we plot the Holweger \& Müller model (1974) for comparison purposes. The temperature stratification of this one-component model of the solar photosphere closely resembles that of our granular component. This result can be understood by considering that the granular component contributes $76 \%$ to the observed intensity profiles. In addition, the Holweger \& Müller model was obtained from measurements of the continuum intensity. In order to convert intensities to temperatures, they implicitly assumed $\mathrm{LTE}^{1}$. Hence, the fact that our LTE inversion leads to similar results should come as no surprise.

\footnotetext{
1 The iron abundance of 7.60 dex adopted by Holweger \& Müller plays a second-order role because it only affects the computation of gas pressures.
} 

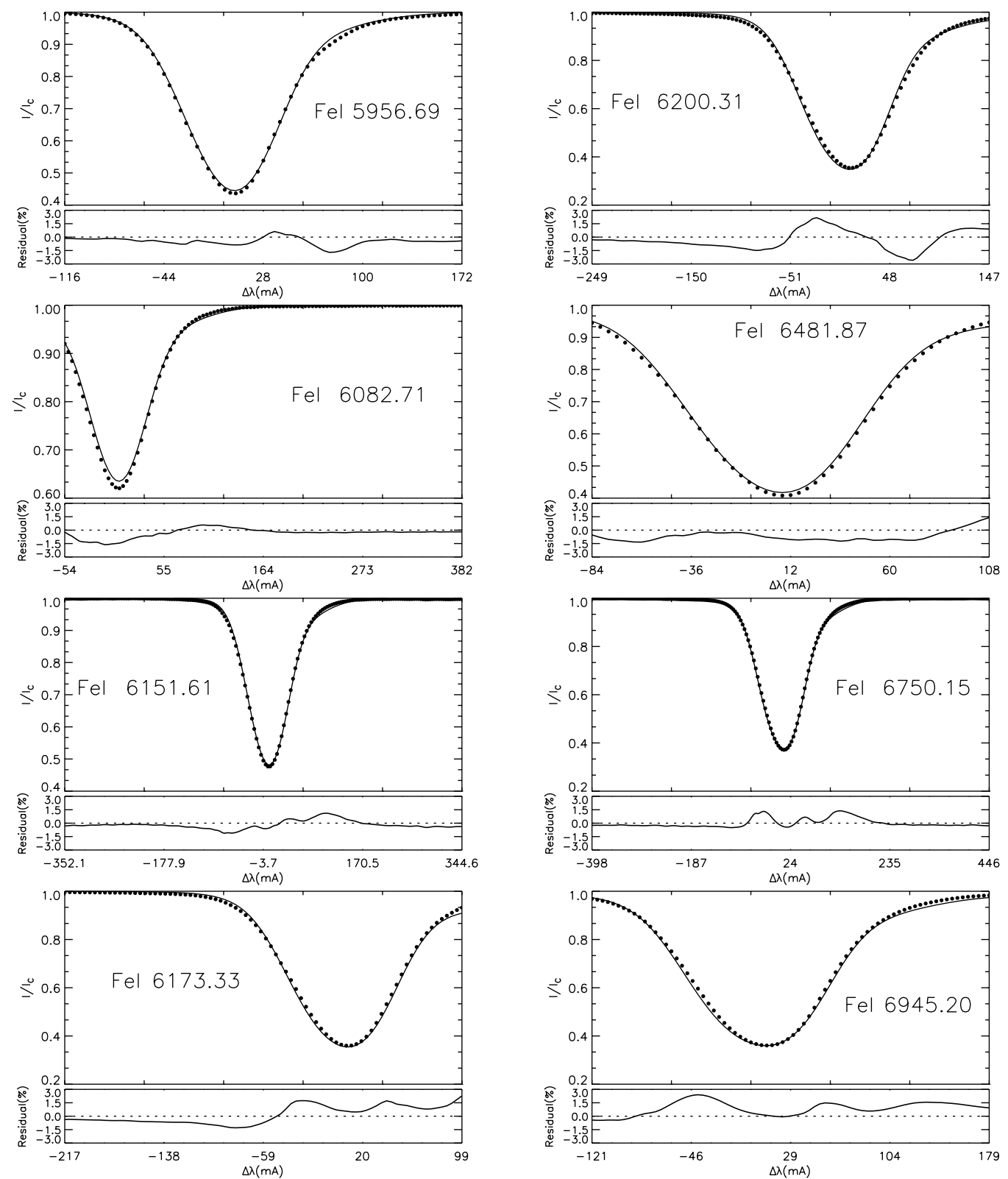

Fig. 2. Comparison between synthetic (solid lines) and observed profiles from the FTS atlas (filled circles) for 8 Fe I spectral lines used in the inversion. The lower panels show the relative residuals of the fit.

\section{Testing the model}

Before accepting the model, it is important to test its ability to reproduce a number of observed features of the solar spectrum. This will tell us about the realism of the model and its usefulness in describing the photosphere of the Sun. To this end we have selected two groups of Fe I lines with the requirements that they do not show obvious blends and that their equivalent widths are smaller than $250 \mathrm{~m} \AA$ (to minimize NLTE effects due to photon losses and scattering). Hereafter, they will be referred to as sets $\mathrm{A}$ and $\mathrm{B}$, respectively.

- Set A: this group contains 663 Fe I lines. Central wavelengths and excitation potentials are taken from Nave et al. (1994). The uncertainty in the laboratory wavelengths is quoted to be $3 \mathrm{~m} \AA$ (or $150 \mathrm{~ms}^{-1}$ ) at
$5000 \AA$. We have used the compilation by O'Brian et al. (1991) for the oscillator strengths. These values are not as accurate as the measurements performed by the Oxford and Hannover-Kiel groups, but the compilation is indeed far more comprehensive. Following Bard \& Kock (1994), an average uncertainty of 0.003 dex in the oscillator strengths can be estimated, with individual values showing a rather large scatter of \pm 0.150 dex. The parameters $\alpha$ and $\sigma$ needed to calculate the collisional broadening have been taken from Barklem et al. (1998) if available. Otherwise, van der Waals broadening is assumed with no extra damping enhancement.

- Set B: the second set contains 119 neutral iron lines. The sources of atomic parameters are the same as for the first set except for the oscillator strengths. Transition probabilities for these lines have been 

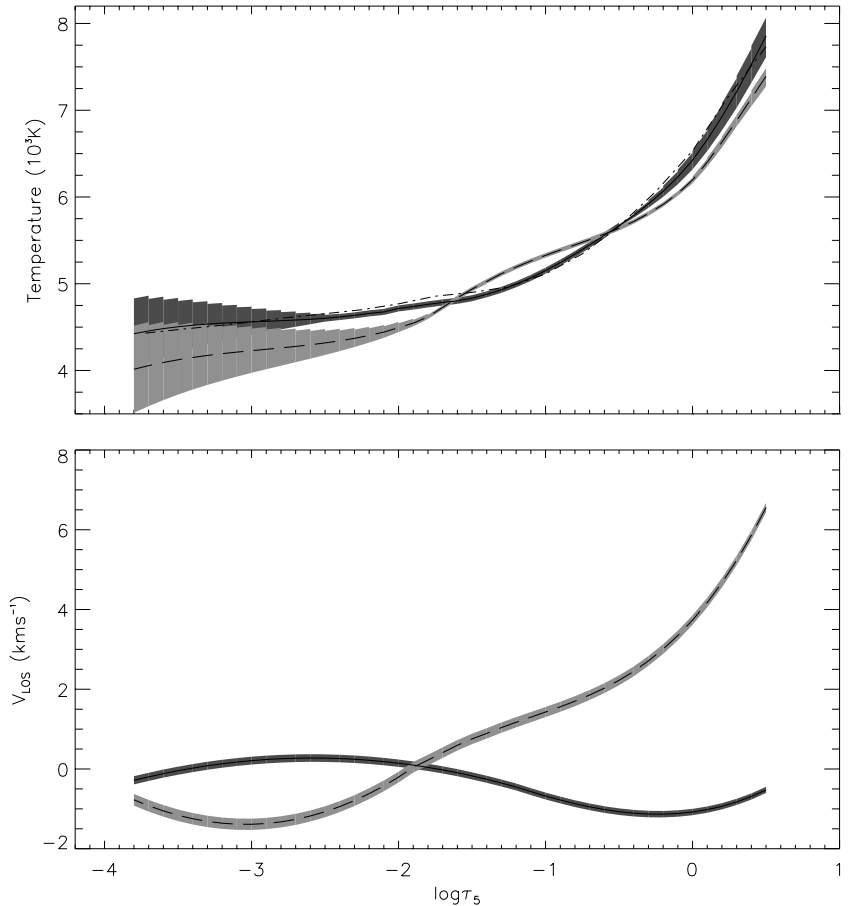

Fig. 3. Two-component model of the quiet Sun inferred from the inversion of the $22 \mathrm{Fe}$ I lines listed in Table 1. The solid and dashed lines indicate the granular and intergranular flows respectively. The filling factor is 0.243. Macroturbulence is $0.86 \mathrm{~km} \mathrm{~s}^{-1}$ for granules and $1.0 \mathrm{~km} \mathrm{~s}^{-1}$ for intergranules. Microturbulence is $0.23 \mathrm{~km} \mathrm{~s}^{-1}$ for granules and $2.05 \mathrm{~km} \mathrm{~s}^{-1}$ for intergranules. Positive velocities indicate downflows. The velocity fields have been corrected for gravitational redshift by substracting $636 \mathrm{~m} \mathrm{~s}^{-1}$ in both components. The shaded areas represent formal errors in the retrieved parameters. The dashdotted line represents the one-component model of Holweger \& Müller (1974).

precisely measured in the laboratory by the Oxford and Hannover-Kiel groups (Blackwell et al. 1982, 1986, 1995a, 1995b; Holweger et al. 1990, 1991, 1995; Bard \& Kock 1991, 1994).

Given the scatter of $\log g f$ values in O'Brian compilation, we will use set A for statistical purposes only. Transition probabilities are far more accurate for lines belonging to set $B$. We thus expect their heights of formation to be established correctly so that the velocity fields and temperatures of the model will be appropriate for the lines to be reproduced. In this regard, set $\mathrm{B}$ will be employed for more quantitative comparisons between observed and predicted behaviors, especially when dealing with observables that are largely dependent on oscillator strengths.

In both cases we have extracted the observed intensity profiles manually from the FTS Atlas. We have synthesized all spectral lines with our two-component model atmosphere and the atomic data mentioned above. Finally, equivalent widths and line shifts have been measured in the observed and synthetic profiles.
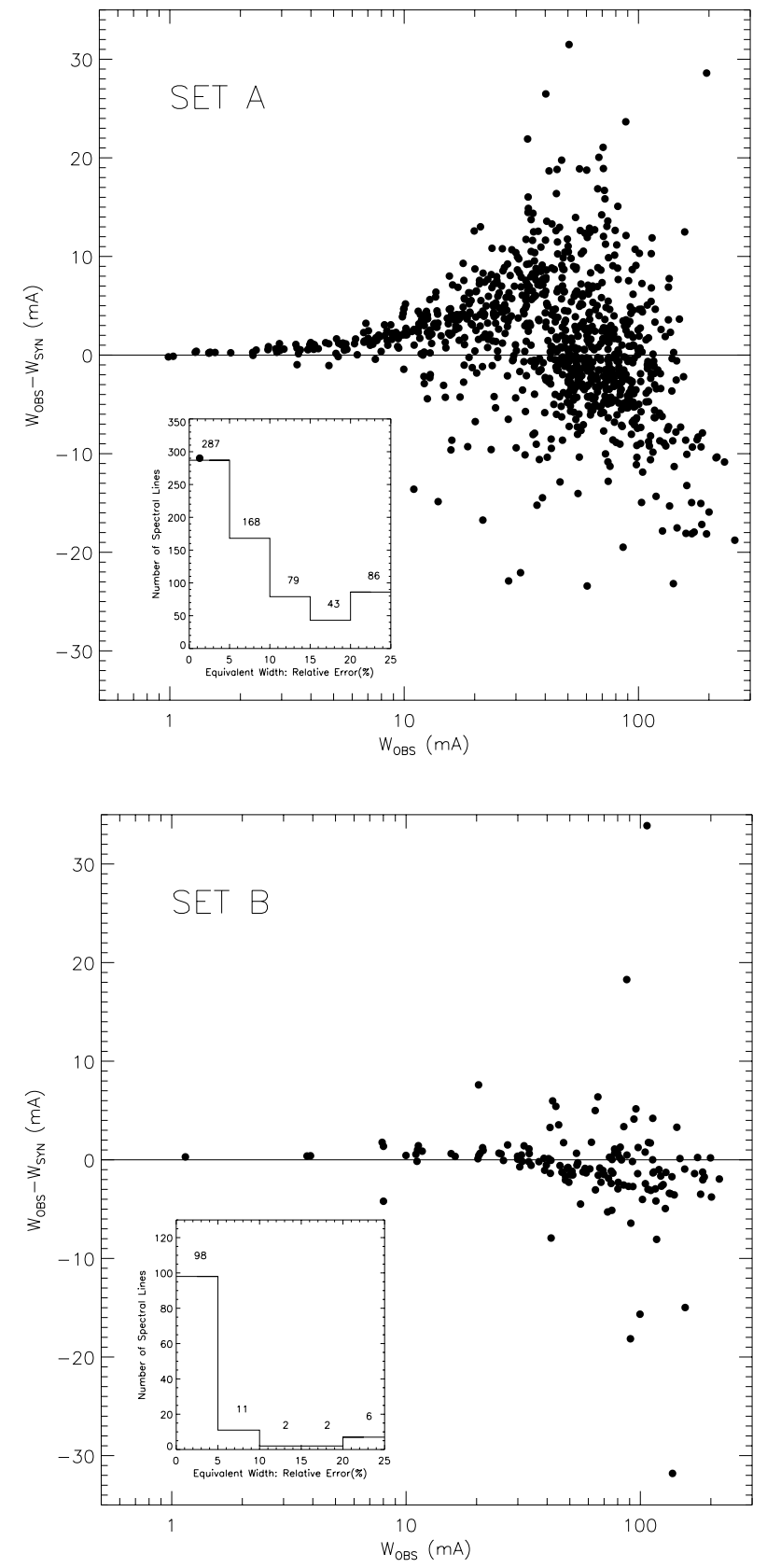

Fig. 4. Difference between observed and computed equivalent widths versus observed equivalents widths for set A (top) and set B (bottom). See text for details.

\subsection{Equivalents widths}

In the upper panel of Fig. 4, a good agreement between observed and predicted equivalent widths for lines belonging to set A can be seen. Intermediate and weak lines (widths $\leq 100 \mathrm{~m} \AA$ ) tend to have greater observed than computed equivalent widths. On the other hand, strong lines (widths $\geq 150 \mathrm{~m} \AA$ ) show the opposite behavior. The mean relative error is $5.6 \%$, with $68 \%$ of the lines having an error smaller than $10 \%$. The comparison of observed and predicted equivalent widths is meant to test the temperature stratifications of our model, but the degree of success of this comparison is strongly dependent on the use of precise oscillator strengths. This is one of the major causes of 


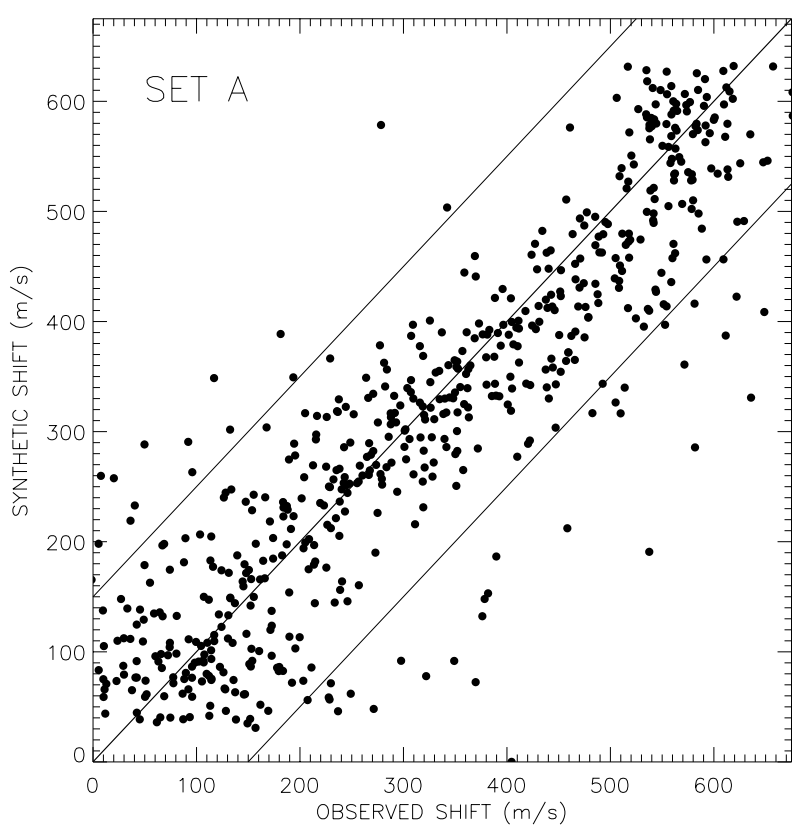

Fig. 5. Predicted versus observed line shifts for $663 \mathrm{Fe}$ I spectral lines (set A). The central line represents a one-to-one correspondence. The solid lines parallel to the central one indicate the $3 \mathrm{~m} \AA$ (or $150 \mathrm{~m} \mathrm{~s}^{-1}$ at $5000 \AA$ ) uncertainty of laboratory measurements by Nave et al. (1994).

the discrepancies found in Fig. 4, especially the deviations in the vertical axis. On the other hand, the observed lines could be affected by blends that are not identified by visual inspection. The influence of weak blends is very difficult to quantify, but in any case we must assume that our lines are contaminated to some degree. This would explain the fact that the observed equivalent width is systematically greater than predicted for weak lines. Intermediate and strong lines are also affected by blends, but proportionally the error induced on the equivalent width is smaller. It is important to note here that, for 136 lines, the $\alpha$ and $\sigma$ parameters required to take into account the new treatment for collisional broadening are not available. This is another source of deviations in the vertical axis, because the predicted equivalent widths of these lines (especially the stronger ones) are underestimated.

In the lower panel of Fig. 4 we represent computed versus observed equivalent widths for lines belonging to set $\mathrm{B}$. It is evident that the agreement is now much better due to the use of laboratory oscillator strengths, giving support to our previous conclusion. In this case, $91 \%$ of the 119 equivalent widths are reproduced with errors smaller than $10 \%$.

\subsection{Line shifts}

Convective motions in stellar atmospheres shift the spectral lines from their laboratory central wavelengths $\lambda_{0}^{\mathrm{LAB}}$. To check whether our model is able to reproduce these convective line shifts in the solar photosphere (and hence the velocity fields originating them) we have calculated the central wavelengths of the observed and synthetic pro-

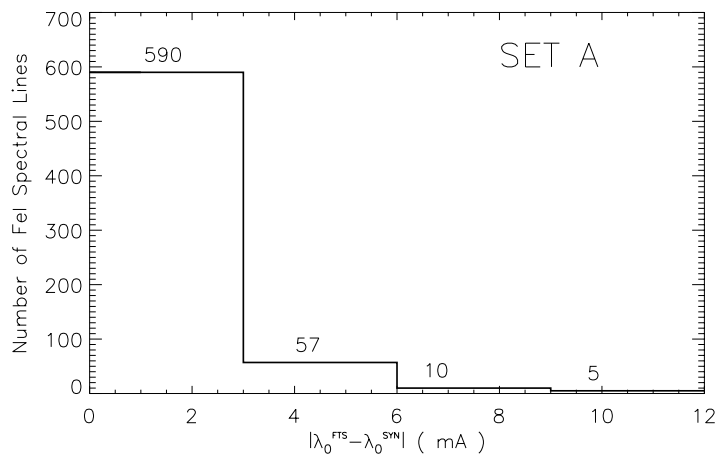

Fig. 6. Histogram of differences between predicted and observed central wavelengths for set A. $89 \%$ of the 663 lines are within the laboratory uncertainty of $3 \mathrm{~m} \AA$.

files, $\lambda_{0}^{\mathrm{OBS}}$ and $\lambda_{0}^{\mathrm{SYN}}$. The results are presented in Fig. 5 for set A. Here we can clearly see that our model is able to reproduce the observed values within the laboratory uncertainty of $3 \mathrm{~m} \AA$ or $150 \mathrm{~m} \mathrm{~s}^{-1}$ at $5000 \AA$. In addition, it is important to note that there are no systematic deviations from a one-to-one correspondence. Along the vertical axis the scatter is due to incorrect oscillator strengths (which change the height of formation of the spectral lines and hence the velocity fields sensed by them) and errors in the velocity fields of the model ${ }^{2}$. Along the horizontal axis, the scatter is due to uncertain laboratory wavelengths. In Fig. 6 we plot a histogram where it can be seen that more than $89 \%$ of the solar central wavelengths are well reproduced within the errors of laboratory measurements. This is the first time a stationary, $1 \mathrm{D}$, two-component model proves to be able to reproduce the observed convective shifts of spectral lines in the solar photosphere. This has important consequences for the practical usage of the model, as it turns out that it is not always necessary to perform highly time--consuming hydrodynamical simulations or use 3-4 component models to properly describe convective motions.

In Fig. 7 we represent the computed versus the observed line shifts for the lines belonging to set B. In Fig. 8 a histogram similar to Fig. 6 is shown. In both figures we see a behavior similar to that found for set A. In this case, however, one source of uncertainty has been eliminated because more accurate transition probabilities were used. This does not seem to improve the agreement between observed and predicted line shifts since, again, $89 \%$ of the values are reproduced within $3 \mathrm{~m} \AA$. This suggests that the main sources of scatter are uncertainties in laboratory wavelengths, which move the data points along the horizontal axis, and possible errors in the velocities of the model.

Convection does not affect all spectral lines equally. It is known that the intensity contrast of the granulation is

\footnotetext{
${ }^{2}$ The uncertainty in the determination of the central wavelength -by means of a fourth-order polynomial fitted to the core $( \pm 25 \mathrm{~mA})$ of each line- is the same for both the observed and synthetic profiles, so it compensates. It is important to note that uncertainties in laboratory wavelengths do not affect the predicted shifts.
} 


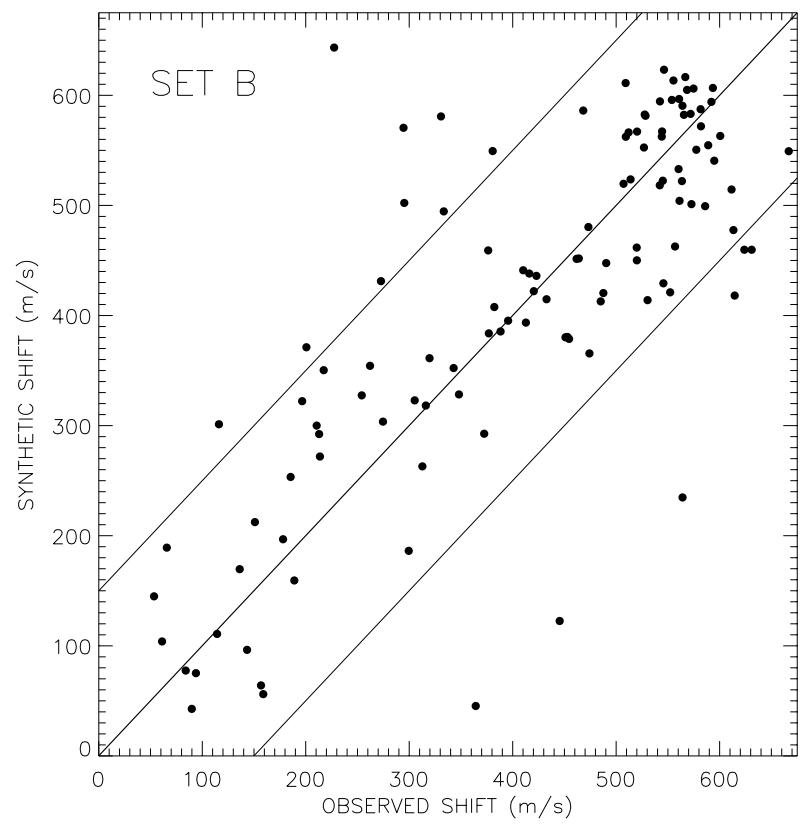

Fig. 7. Predicted versus observed line shifts for 119 Fe I spectral lines (set B). The central line indicates a one-to-one correspondence. The solid lines parallel to the central one indicate the $3 \mathrm{~m} \AA$ or $150 \mathrm{~m} \mathrm{~s}^{-1}$ at $5000 \AA$ uncertainty of laboratory measurements by Nave et al. (1994).

much larger in the continuum (deep layers) than in the line cores (high layers). This means that convection does not penetrate from the convection zone up to the higher photospheric layers. As a consequence, spectral lines whose cores are formed in deep layers (i.e., weak lines) are more affected by convection, which leads to a convective blueshift due to the greater contribution of granules. On the other hand, lines whose cores are formed in high layers (i.e., strong lines) are not influenced by such motions because the line-of-sight velocity vanishes. In addition, all spectral lines are affected by the solar gravitational field which causes them to be redshifted by $636 \mathrm{~m} \mathrm{~s}^{-1}$. It is important to check whether this behavior is reproduced by our model.

In Fig. 9 (top) we have plotted the line shifts as a function of equivalent widths for the 663 spectral lines (set A) extracted from the FTS atlas. It can be seen that the stronger the line considered (increasing equivalent widths), the smaller the convective blueshift. For very strong lines (formed in high layers) convection does not influence the profiles, and the only source of shift is the gravitational redshift. The scatter in the vertical axis arises mainly from errors in laboratory wavelengths, while the scatter in the horizontal axis is due to the different atomic parameters of the lines and the possibility of weak blends which go unnoticed on visual inspection. The central panel of Fig. 9 represents the same parameters for the 663 lines of set A synthesized with our model. It is clear that the general trend is well reproduced. Nevertheless, the saturation at $\simeq 600 \mathrm{~ms}^{-1}$ begins at an equivalent width of $\simeq 90 \mathrm{~m} \AA$ for the observed lines, while the synthetic lines show the same saturation at $\simeq 110 \mathrm{~m} \AA$, perhaps as a con-

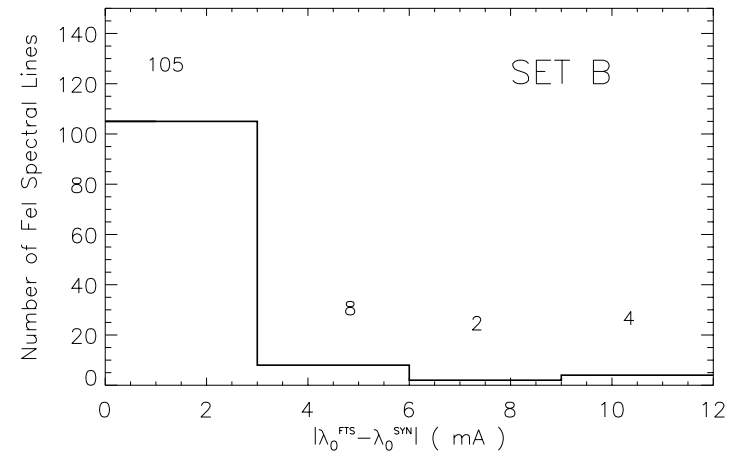

Fig. 8. Histogram of differences between predicted and observed central wavelengths for set B. $89 \%$ of the 119 lines are within the laboratory uncertainty of $3 \mathrm{~mA}$.

sequence of our overestimation of equivalent widths for strong lines (see Fig. 4, top). Now the sources of scatter in the vertical axis are errors in transition probabilities and in the velocity fields of the model, while the scatter in the horizontal axis is due to errors in transition probabilities and temperatures.

Figure 9 (bottom) contains the same plot for set B. The general behavior is again well reproduced. Although the small number of lines considered in this case makes any comparison difficult, it appears that using more accurate atomic parameters does not improve the agreement between predictions and observations. From this we believe that the dominant source of scatter in the predictions is errors in the velocities and temperatures of the model, rather than errors in transition probabilities.

\subsection{Limb darkening}

While the previous tests have concerned mostly velocity fields, this test is conceived for checking the thermal structure of the model. At a given frequency photons come from layers where the optical depth at that frequency is unity. As we move from the center of the disk $(\mu=\cos \theta=1)$ to the limb $(\mu=0)$, such layers are located at a higher geometrical height, where the temperature is lower. As a consequence, the continuum intensity decreases towards the limb. We have computed the continuum intensity at different wavelengths and different positions on the disk and compared our predictions with the polynomials fitted by Neckel \& Labs (1994).

Figure 10 shows the results of this test along with the predictions of the Holweger \& Müller model and the Harvard Smithsonian Reference Atmosphere (HSRA, Gingerich et al. 1971). The center-to-limb variation resulting from our model is in excellent agreement with the observed behavior, except for angles near the limb where the continuum intensity seems to be overestimated. Among other reasons, this could be due to erroneous temperatures in high layers or to our neglecting the lateral interaction between the two atmospheric components, which can be expected to become increasingly important towards the limb. Nevertheless, our model turns out to be superior to the widely used Holweger \& Müller one- 

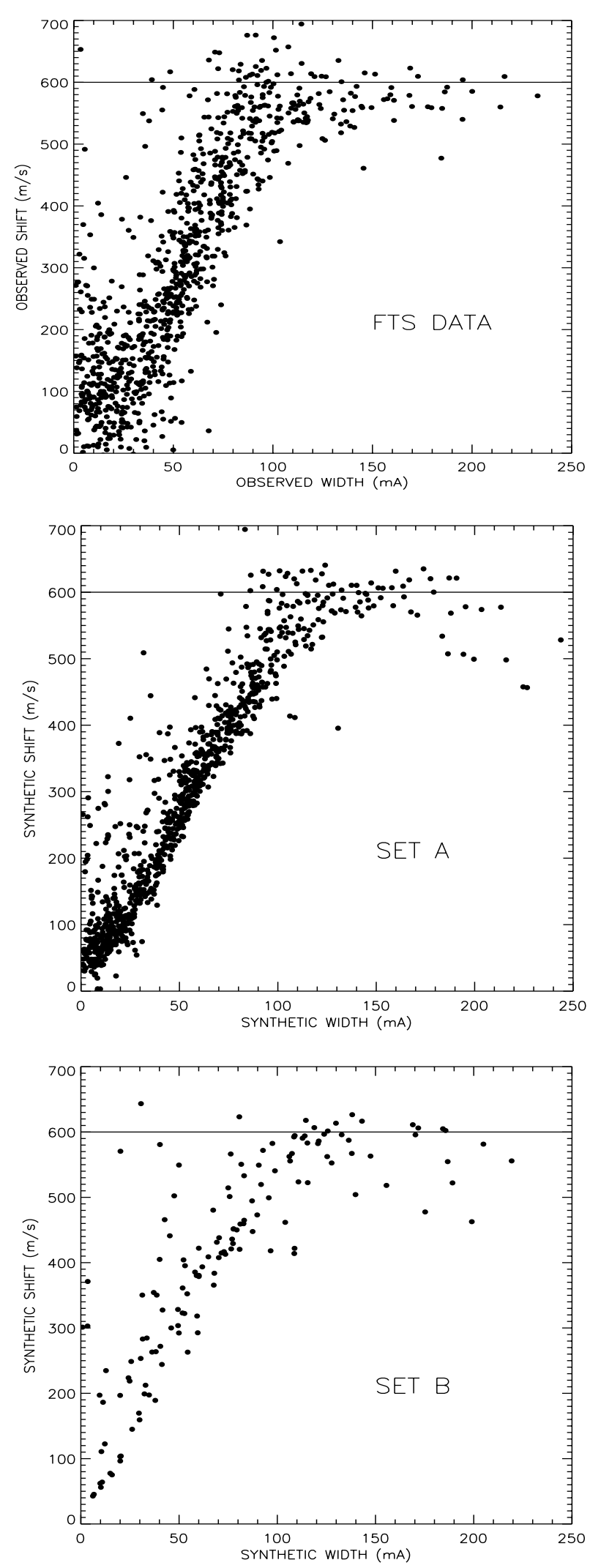

Fig. 9. Line shifts versus equivalent widths. Top: observed lines. This plot is similar to that obtained by Allende Prieto et al. (1997). Middle: synthetic lines belonging to set A. Bottom: synthetic lines belonging to set B. component model of the solar photosphere. It is also superior to the HSRA model for heliocentric angles smaller than about $66^{\circ}(\mu \simeq 0.4)$, and of similar quality for larger angles. This is remarkable because the HSRA model was constructed from measurements of the center-to-limb variation of the continuum intensity.

\section{Determination of atomic data}

Once the degree of realism of the model has been demonstrated by its ability to reproduce several important features of the solar spectrum, it is possible to use it for determining accurate line transition parameters for spectral lines lacking laboratory measurements. In this section we explore the feasibility of this idea and quantify the accuracy of the determined atomic parameters. Since the following analysis is for illustration purposes only, we will concentrate on the estimation of oscillator strengths. The feasibility of deriving reliable central wavelengths has been shown by Bellot Rubio et al. (1999).

\subsection{Neutral iron}

Our two-component model is applied here to estimate oscillator strengths for the 119 Fe I lines of set B. We start by synthesizing the 119 lines using oscillator strengths from Thévenin $(1989,1990)$ instead of the laboratory values adopted in the previous section. Oscillator strengths compiled by Thévenin were derived from analysis of the solar spectrum. Due to the use of one-component model atmospheres and the fitting of equivalent widths instead of full intensity profiles, these values are not as accurate as laboratory measurements. Consequently, the synthesized intensity profiles will differ greatly from the observed ones. Since we suppose our model to be realistic, any difference between observed and synthesized profiles will be explained as due to incorrect transition probabilities which we will change iteratively until the synthetic profiles match the observed ones (see Fig. 11 for an illustration of the different profile shapes corresponding to different oscillator strengths). Figure 12 (top) represents the differences between the laboratory and Thévenin oscillator strengths, $\Delta=\log g f_{\mathrm{FUR}}-\log g f_{\mathrm{THE}}$, versus the laboratory oscillator strength $\log g f_{\mathrm{FUR}}$. As can be seen, the agreement between Thévenin and laboratory measurements is poor due to the aforementioned reasons. The mean difference is $\Delta=0.133$, while the scatter is $\sigma=0.306$. Using our model to correct Thévenin's values (Fig. 12, bottom), the differences between the new oscillator strengths and those from laboratory are greatly reduced. Now $\Delta=0.038$ and $\sigma=0.076$. This means that we can measure oscillator strengths with much more accuracy than that achieved by May et al. (1974), Thévenin $(1989,1990)$ and O'Brian (1991) (see Bard \& Kock 1994 for details). Our method is still not as accurate as laboratory measurements, but we can put a high degree of confidence on it. Indeed, $62 \%$ of the 119 lines differ from the laboratory values by less than 0.06 dex, which is the mean difference between the oscillator strengths provided by the Oxford and Hannover-Kiel groups, and $82 \%$ of the 119 lines can be measured within 


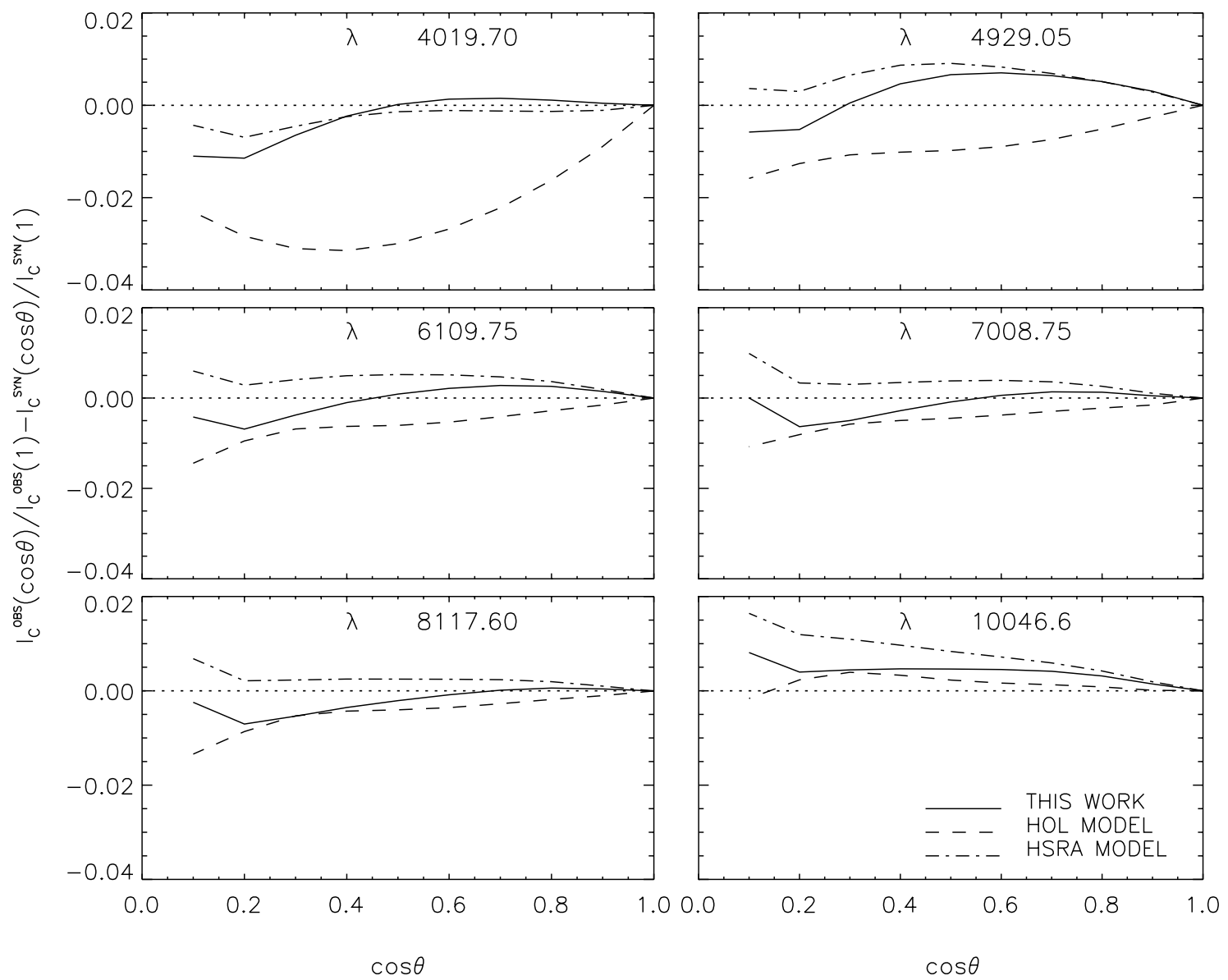

Fig. 10. Center-to-limb variation of the continuum intensity $I_{\mathrm{c}}$ predicted by our model (solid lines), Holweger \& Müller model (dashed lines) and Harvard Smithsonian Reference Atmosphere of Gingerich et al. (1971, dash-dotted lines) at different wavelengths in the optical spectrum. The observed behavior $I_{\mathrm{c}}^{\mathrm{obs}}(\mu) / I_{\mathrm{c}}^{\mathrm{obs}}(1)$ is given by the polynomials fitted by Neckel \& Labs (1994).

the laboratory uncertainty (0.11 dex according to Bard \& Kock 1994). For comparison, only $35 \%$ of Thévenin values are within the laboratory uncertainty.

\section{2. lonized iron and other species}

In the last years, the study of the solar iron abundance using Fe II lines instead of neutral iron has gained attraction because they show smaller departures from LTE. It is then desirable to have accurate line transition parameters for Fe II. Unfortunately this is not the case. The uncertainties of the best oscillator strengths and central wavelengths are $\simeq 0.2$ dex and $\simeq 20 \mathrm{~m} \AA$, respectively. The situation is even worse for other important elements such as Si, Cr and Ti. Despite our model has been obtained from the inversion of Fe I lines, it should be possible to use it to determine line transition parameters for other elements and ions if the thermal and velocity stratifications of the model are realistic.

To this end we have compiled a list of spectral lines of elements other than iron including their first ionization stages. Again the only restriction applied is that these spectral lines must have accurate oscillator strengths measured in the laboratory, so that we can compare our pre- dictions. Since it is extremely difficult to build a large list if we impose this constraint, we have taken the list used by Allende Prieto et al. $(2001)^{3}$ in their inversions. Although the oscillator strengths seem to be accurate we cannot say the same about the central wavelengths (the values given by Allende Prieto et al. come from the flux atlas of the Sun by Kurucz et al. 1984), so we will not study the line shifts produced by convection. In any case, it would be possible to determine accurate central wavelengths for these lines using the strategy explained in Sect. 5.1.

The final list contains 9 Si I, 6 Ca I, 10 Ti I, 7 Cr I, $15 \mathrm{Fe}$ II, and $3 \mathrm{Ti}$ II lines. Prior to the determination of oscillator strengths we have computed their equivalent widths using the laboratory transition probabilities and abundances given by Thévenin (7.50 dex for Si, 6.36 for $\mathrm{Ca}, 4.88$ for $\mathrm{Ti}$, and 5.61 for $\mathrm{Cr}$ ). In Fig. 13 we show the comparison between predicted and observed equivalent widths. It turns out that $80 \%$ of the observed values are reproduced with relative errors smaller than $10 \%$, and therefore the degree of success of this test is similar to that of the 119 Fe I lines with very precise oscillator strengths

3 The sources of data in this list are Kroll \& Kock (1987), Holweger et al. (1990), Hannaford et al. (1992) and Schnabel et al. (1999). 


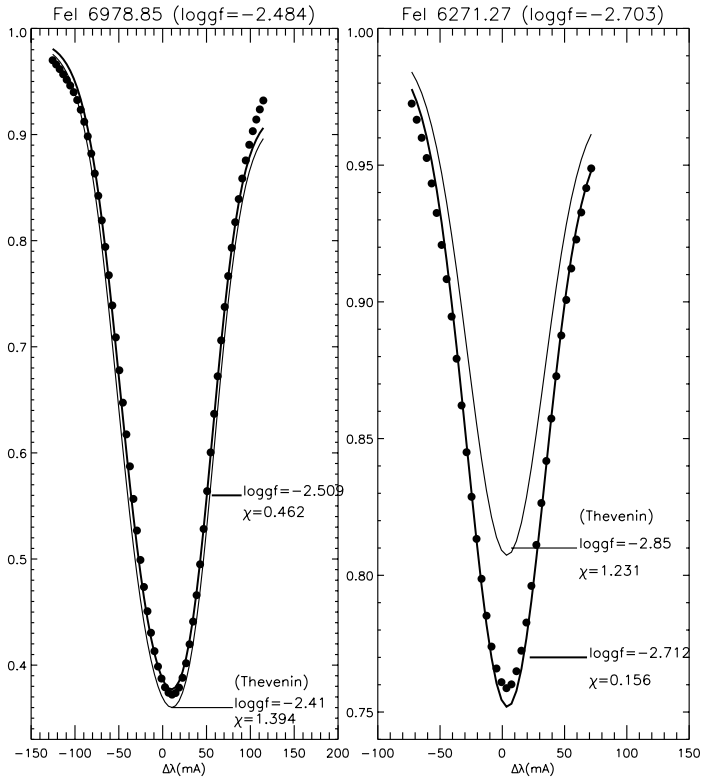

Fig. 11. Two examples of the determination of transition probabilities for neutral iron lines. Dots represent intensity profiles extracted from the FTS Atlas. The thin solid lines represent the synthesized profiles using Thévevin $(1989,1990)$ oscillator strengths. These values are changed until the merit function $\chi=\sum_{k}\left|I_{\mathrm{FTS}}\left(\lambda_{k}\right)-I_{\mathrm{SYN}}\left(\lambda_{k}\right)\right|$ reaches a minimum. The final synthetic profiles are represented by thick solid lines. The oscillator strengths of these two spectral lines have been measured in the laboratory. Fe I $6978.85 \AA$ (left): $\log g f_{\mathrm{FUR}}=-2.484$. Fe I 6271.27 (right): $\log g f_{\mathrm{FUR}}=-2.703$.

considered in Sect. 4.1. On the other hand, only $53 \%$ of the equivalent widths are reproduced with a relative error smaller than $5 \%$, perhaps as a consequence of the fact that laboratory measurements for these elements are not as accurate as for neutral iron.

The determination of oscillator strengths using our two-component model has been carried out as explained before. In Fig. 14 (top) we plot the difference between laboratory oscillator strengths and Thevenin's values. The mean deviation is $\Delta=0.150$ and the scatter is $\sigma=0.09$. In Fig. 14 (bottom), we plot the difference between laboratory oscillator strengths and those derived from our two-component model. The mean deviation in this case is $\Delta=-0.001$ and the scatter is $\sigma=0.06$.

The improvement is very remarkable. Now $72 \%$ of the 50 lines have differences between the new and laboratory oscillator strengths smaller than $0.06 \mathrm{dex}$, and 98\% have differences smaller than 0.11 dex. Therefore we have extended our predictive capability to other neutral and singly ionized species using a two-component model obtained from the inversion of Fe I spectral lines only. Clearly, this excellent result could not have been achieved if the thermal structure of the model were wrong.

\section{NLTE effects}

From forward modeling we know that almost all neutral iron lines show deviations from LTE to a greater or lesser extent. This of course has an impact on the emergent

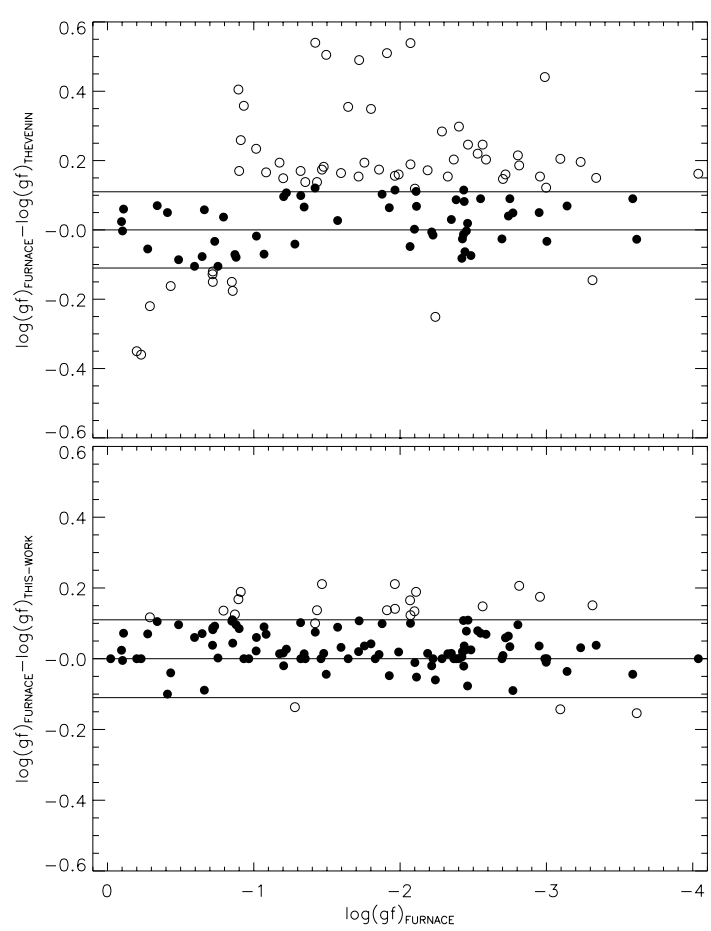

Fig. 12. Top: differences between laboratory (Oxford and Hannover-Kiel groups) and solar oscillator strengths from Thévenin $(1989,1990)$ for 119 neutral iron lines. The mean difference is $\Delta=0.133$ and the scatter is $\sigma=0.306$. Empty circles represent differences larger than the maximum laboratory uncertainty of 0.11 dex. Filled circles indicate differences within the laboratory uncertainty. Bottom: differences between laboratory oscillator strengths and those predicted by our twocomponent model for 119 neutral iron lines. The mean difference is $\Delta=0.038$ and the scatter is $\sigma=0.076$.

intensity profiles. A very detailed investigation of these effects has recently been presented by Shchukina \& Trujillo Bueno (2001). Neglecting horizontal radiation transfer, the most important NLTE mechanism is overionization of iron due to the near-UV radiation field (Athay \& Lites 1972), which significantly underpopulates the Fe I atomic levels and slightly overpopulates the Fe II levels. For Fe I, departure coefficients are generally smaller than unity. This decreases the line opacities with respect to their LTE values. As a result, the spectral lines are formed in deeper layers than prescribed by LTE populations and, because of the prevailing higher temperatures, they are weaker than their LTE counterparts. The line source function for Fe I lines is also modified with respect to LTE. Lines of intermediate and high lower excitation potentials have smaller line source functions than in LTE. The stronger the line, the more important the deviation from LTE as a consequence of photon losses (Bruls et al. 1992). Modifications of the line source function generally lead to spectral lines that are stronger than in LTE.

Shchukina \& Trujillo Bueno (2001) point out that these two effects (changes in the line opacity and the line source function) work in opposite directions so that they compensate to some extent, implying that intensity profiles computed by assuming LTE do not differ very 


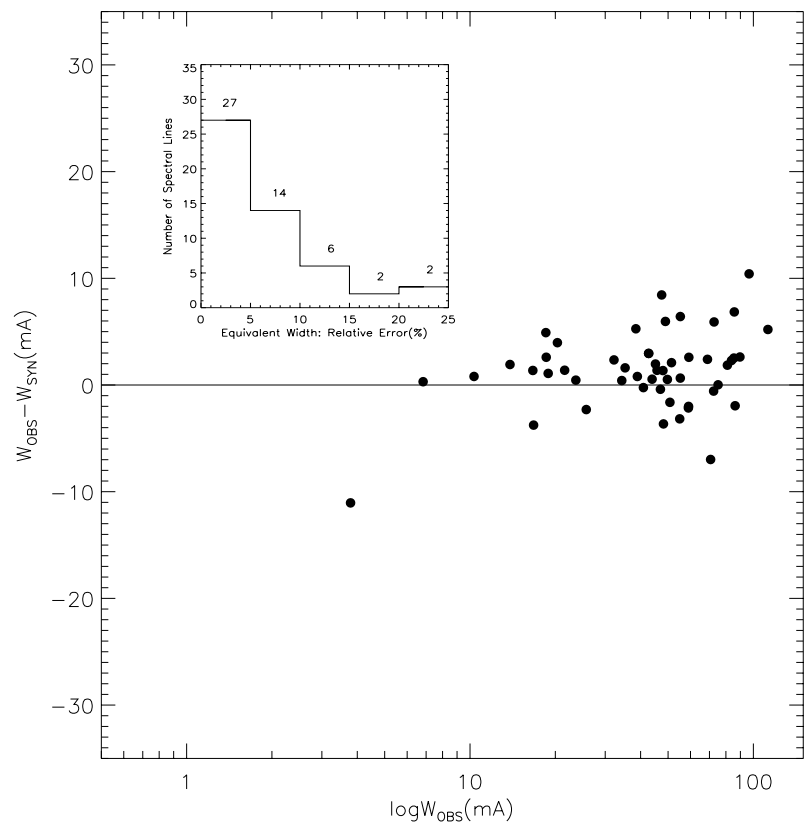

Fig. 13. Difference between observed and predicted equivalent widths versus observed equivalent widths for 50 spectral lines of various elements (see text for details). The vertical scale is the same as in Fig. 4.

much from those synthesized by NLTE codes. Given the similar profiles resulting from LTE and NLTE spectral syntheses, with the latter being slightly weaker on average, Shchukina \& Trujillo Bueno concluded that it is possible to obtain good fits to observed lines assuming LTE if the iron abundance is lowered with respect to that needed to reproduce the same profiles under NLTE conditions. They quote an average difference of $0.07 \mathrm{dex}$. This might explain the success of our LTE inversion, which uses an iron abundance of $7.46 \mathrm{dex}$, while the NLTE abundance derived by Shchukina \& Trujillo Bueno (2001) turns out to be equal to the meteoritic abundance value $(7.50 \pm 0.10$ dex).

Although each line behaves differently (low excitation potential lines show the largest departures from LTE, for example), several general tendencies can be expected from the above considerations. First, due to the larger line opacities resulting from LTE atomic populations, spectral lines will tend to form higher in the photosphere under LTE conditions. Second, we expect LTE temperatures to be slightly larger than NLTE temperatures (by 50$100 \mathrm{~K}$ ) in the middle photosphere because of the NLTE line source function deficits. According to Shchukina \& Trujillo Bueno, such effects are important in granules, but not in intergranules. At least in part, they may explain the flat temperature stratifications of our model (see Fig. 4).

Since each transition is influenced by NLTE effects in a different way (even in opposite ways), it is important to use lines spanning a wide range of atomic parameters for minimizing systematic trends that may arise in an LTE treatment. In order to check whether our model is much affected by the neglect of NLTE effects, we have analyzed the errors of its predictions as a function of excitation

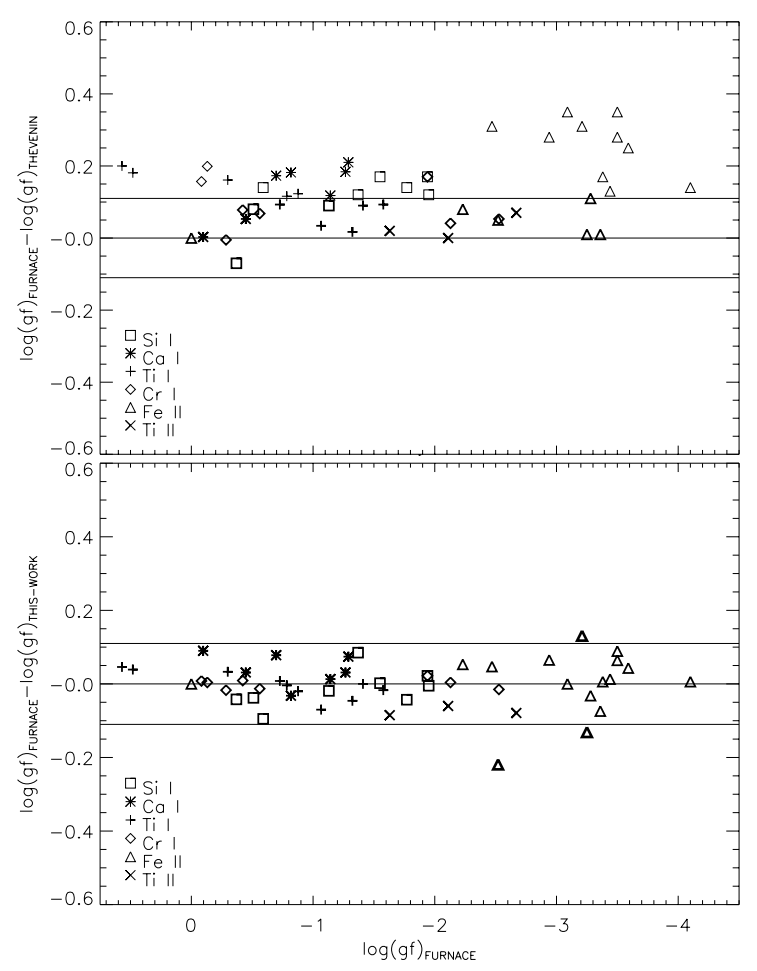

Fig. 14. Top: difference between laboratory oscillator strengths (Oxford and Hannover-Kiel groups) and values from Thévenin $(1989,1990)$ for 50 spectral lines of various elements. The mean difference is $\Delta=0.150$ and the scatter is $\sigma=0.09$. Bottom: difference between laboratory oscillator strengths (Oxford and Hannover-Kiel groups) and those predicted by our two-component model for the same spectral lines. The mean difference is $\Delta=-0.001$ and the scatter is $\sigma=0.06$.

potentials and oscillator strengths. We do not find any systematic trend, suggesting that NLTE effects are small compared to other sources of error. In part, this has been achieved by ignoring spectral lines with equivalent widths greater than $250 \mathrm{~m} \AA$, which are more prone to photon losses. Another reason is that the model has not been extended to the lower chromosphere $\left(\log \tau_{5} \epsilon[-4,-6]\right)$, where departure coefficients start to deviate markedly from unity. Unfortunately, by doing so we limit the applicability of the model to relatively weak spectral lines. It would be desirable to extend the model to higher layers in order to include lines that cannot be considered at present (mainly strong photospheric lines). However, as we move from the high photosphere to the lower chromosphere, NLTE effects become more and more important for $\mathrm{Fe} \mathrm{I}$.

One possibility to extend the model is to use Fe II lines in the inversion. Including Fe II has the advantage that it is less sensitive to NLTE effects, so in principle it could be possible to perform a full LTE treatment. Although with different purposes, Allende Prieto et al. (2001) and Frutiger et al. (2000) have used spectral lines of both neutral and ionized species in their inversions. While Allende Prieto et al. claim that it is better to invert lines of neutral and ionized elements in order to break down the temperature-abundance degeneracy, Frutiger et al. did so 
because of their different sensitivity to temperature. It is remarkable that Frutiger et al. obtained poor agreement between observed and synthetic profiles using Fe I and Fe II in their LTE inversions. Similarly to these authors, we have carried out inversions where Fe I and Fe II lines were considered simultaneously and, again, poor agreements were found. Due to the overionization of iron by ultraviolet radiation (the most important NLTE effect), the temperature stratification in high layers becomes inconsistent in a LTE treatment as soon as we try to invert lines from both ionization stages. This of course confirms the previous findings by Frutiger et al., although they did not identify this as a pure NLTE effect and included Fe II in their analysis. Because of such an inconsistency, it is clear that using Fe I and Fe II lines in an LTE inversion would not allow us to increase the degree of realism of the model in the upper layers.

Another possibility is to perform LTE inversions of Fe II lines only. While this seems appropriate at first sight, one must bear in mind that the uncertainties in atomic parameters are very large for ionized iron. This has important consequences in practice, as it might be impossible to find temperature and velocity stratifications capable of reproducing the observed profiles of all spectral lines considered. More important is the fact that one would need very strong Fe II lines to extend the model to higher layers. Such lines would have a scattering source function, which would again rule out LTE treatments. Thus, a full NLTE analysis appears to be necessary for extending the model to the lower chromosphere.

\section{Conclusions}

We have obtained a 1D, two-component model of the solar photosphere from a LTE inversion of 22 neutral iron lines from the quiet sun (FTS atlas at disk center spatially and temporally averaged). These two components can be visualized as granular and intergranular components. The model is able to reproduce the observed line shifts and equivalent widths of two large compilations of neutral iron lines. The degree of success of these tests, except perhaps those concerning convective motions, seems to be strongly dependent upon the accuracy of the atomic parameters employed. Additionally, we have compared the predictions of the model with the observed center-to-limb variation of the continuum intensity. This test shows that our model is superior to the widely used Holweger \& Müller onecomponent model atmosphere and similar to, if not better than, the Harvard Smithsonian Reference Atmosphere of Gingerich et al. (1971).

For the first time, a two-component model has proved to be able to reproduce very different observational features of the solar spectrum. This suggests that the temperature and velocity stratifications of the model are essentially correct. Our results indicate that there is no need to use 3 or 4 -component models or perform 3D hydrodynamical simulations for a reliable description of the effects of convective motions. We also have shown that the model derived in this paper can be used to infer accurate values of central wavelengths and transition probabilities for neutral iron and other species within the accuracy of the best laboratory measurements. The main advantage of using the Sun as a laboratory is that we are not restricted to transitions excitable on Earth.

In view of the increasing need of accurate atomic data for many astrophysical investigations, we plan to use our model for evaluating line transition parameters for a large list of spectral lines. The implementation of the corresponding response functions in the inversion code will improve not only the speed but also the reliability of this process. It is however desirable to perform a NLTE analysis in order to reduce the uncertainties and extend the applicability of our treatment to very strong lines. Other applications of the model include abundance determination, calibration of stellar spectra, and description of convective motions in the Sun and Sun-like stars.

Solar abundance studies have been traditionally carried out by fitting equivalent widths with one-component models. Since these models do not consider convective motions, they can only produce symmetric profiles. Thus, systematic errors in the predicted equivalent widths are very likely, and these may translate into erroneous abundance values. This has been identified by Blackwell et al. (1995a, 1995b) and Holweger et al. (1995) as one of the major causes of the discrepancy maintained by the Oxford and Hannover-Kiel groups concerning the solar iron abundance. Now that two-component models of the solar photosphere are available, this source of error can be minimized.

In stellar astrophysics it is not possible to derive model atmospheres from continuum intensity measurements since we observe flux instead of intensity. Therefore, models have been obtained directly from the inversion of spectral lines. Many recent abundance determinations in cool stars use one-component model atmospheres (e.g., Allende Prieto et al. 1998, 2001). Frutiger et al. (2001) made an important progress by deriving multi-component models from the flux of sun-like stars. Unfortunately, no further tests were made to support the reliability of such models or check whether they are able to describe convective motions. In this paper we have demonstrated that a simple two-component model provides a good representation of the photosphere of the closest cool star, the sun. Certainly, the procedure outlined in this work (determination of a two-component model atmosphere from inversion of spectral lines, use of the best atomic parameters available, and fitting of observed profiles) can be followed for accurate measurements of abundances in other cool stars. This will improve our knowledge of the chemical evolution of the galaxy as well as its cosmological implications.

Acknowledgements. Very special thanks to Paul Barklem for his help during the implementation of the quantum-mechanical description of the collisional broadening, and to Manolo Collados and Basilio Ruiz Cobo for a careful reading of the manuscript. We also thank Carlos Allende Prieto for his help and long-lasting interest in this project. 


\section{Appendix A: Two-component model of the quiet solar photosphere}

Table A.1. Granular component derived from the inversion. Microturbulence is equal to $0.23 \mathrm{~km} \mathrm{~s}^{-1}$ and macroturbulence is $0.85 \mathrm{~km} \mathrm{~s}^{-1} \cdot \tau_{5}$ stands for continuum optical depth at $5000 \AA, z$ for geometrical height, $T$ for temperature, $P_{\mathrm{e}}$ for electron pressure, $P_{\mathrm{g}}$ for gas pressure, $\rho$ for density, and $v_{\mathrm{LOS}}$ for line-of-sight velocity. Negative velocities indicate upflows. The gravitational redshift $\left(636 \mathrm{~m} \mathrm{~s}^{-1}\right)$ has been subtracted. $z=0 \mathrm{~km}$ corresponds to $\tau_{5}=1$.

\begin{tabular}{|c|c|c|c|c|c|c|}
\hline $\log \tau_{5}$ & $z(\mathrm{~km})$ & $T(\mathrm{~K})$ & $P_{\mathrm{e}}\left(\right.$ dyn $\left.\mathrm{cm}^{-2}\right)$ & $P_{\mathrm{g}}\left(\right.$ dyn $\left.\mathrm{cm}^{-2}\right)$ & $\rho\left(\mathrm{g} \mathrm{cm}^{-3}\right)$ & $v_{\mathrm{LOS}}\left(\mathrm{km} \mathrm{s}^{-1}\right)$ \\
\hline 1.0 & -55.1 & 9400 & $4.799 \mathrm{E}+03$ & $1.808 \mathrm{E}+05$ & $3.013 \mathrm{E}-07$ & 0.76 \\
\hline 0.9 & -51.9 & 9094 & $3.479 \mathrm{E}+03$ & $1.780 \mathrm{E}+05$ & $3.067 \mathrm{E}-07$ & 0.44 \\
\hline 0.8 & -48.3 & 8792 & $2.473 \mathrm{E}+03$ & $1.750 \mathrm{E}+05$ & $3.119 \mathrm{E}-07$ & 0.15 \\
\hline 0.7 & -44.5 & 8490 & $1.716 \mathrm{E}+03$ & $1.718 \mathrm{E}+05$ & $3.170 \mathrm{E}-07$ & -0.10 \\
\hline 0.6 & -40.4 & 8184 & $1.153 \mathrm{E}+03$ & $1.682 \mathrm{E}+05$ & $3.219 \mathrm{E}-07$ & -0.33 \\
\hline 0.5 & -35.9 & 7855 & $7.285 \mathrm{E}+02$ & $1.641 \mathrm{E}+05$ & $3.273 \mathrm{E}-07$ & -0.52 \\
\hline 0.4 & -30.6 & 7528 & $4.446 \mathrm{E}+02$ & $1.594 \mathrm{E}+05$ & $3.317 \mathrm{E}-07$ & -0.69 \\
\hline 0.3 & -24.6 & 7213 & $2.656 \mathrm{E}+02$ & $1.539 \mathrm{E}+05$ & $3.342 \mathrm{E}-07$ & -0.82 \\
\hline 0.2 & -17.6 & 6925 & $1.599 \mathrm{E}+02$ & $1.474 \mathrm{E}+05$ & $3.335 \mathrm{E}-07$ & -0.93 \\
\hline 0.1 & -9.43 & 6660 & $9.728 \mathrm{E}+01$ & $1.400 \mathrm{E}+05$ & $3.293 \mathrm{E}-07$ & -1.02 \\
\hline 0.0 & 0.00 & 6424 & $6.085 \mathrm{E}+01$ & $1.316 \mathrm{E}+05$ & $3.209 \mathrm{E}-07$ & -1.08 \\
\hline-0.1 & 10.6 & 6231 & $4.068 \mathrm{E}+01$ & $1.224 \mathrm{E}+05$ & $3.078 \mathrm{E}-07$ & -1.12 \\
\hline-0.2 & 22.4 & 6065 & $2.846 \mathrm{E}+01$ & $1.128 \mathrm{E}+05$ & $2.913 \mathrm{E}-07$ & -1.13 \\
\hline-0.3 & 35.0 & 5920 & $2.070 \mathrm{E}+01$ & $1.030 \mathrm{E}+05$ & $2.726 \mathrm{E}-07$ & -1.13 \\
\hline-0.4 & 48.5 & 5788 & $1.547 \mathrm{E}+01$ & $9.336 \mathrm{E}+04$ & $2.526 \mathrm{E}-07$ & -1.11 \\
\hline-0.5 & 62.6 & 5669 & $1.190 \mathrm{E}+01$ & $8.399 \mathrm{E}+04$ & $2.321 \mathrm{E}-07$ & -1.07 \\
\hline-0.6 & 77.2 & 5558 & $9.348 \mathrm{E}+00$ & $7.511 \mathrm{E}+04$ & $2.117 \mathrm{E}-07$ & -1.02 \\
\hline-0.7 & 92.2 & 5447 & $7.415 \mathrm{E}+00$ & $6.682 \mathrm{E}+04$ & $1.921 \mathrm{E}-07$ & -0.95 \\
\hline-0.8 & 107 & 5341 & $5.983 \mathrm{E}+00$ & $5.920 \mathrm{E}+04$ & $1.736 \mathrm{E}-07$ & -0.87 \\
\hline-0.9 & 122 & 5243 & $4.904 \mathrm{E}+00$ & $5.229 \mathrm{E}+04$ & $1.562 \mathrm{E}-07$ & -0.78 \\
\hline-1.0 & 137 & 5151 & $4.071 \mathrm{E}+00$ & $4.610 \mathrm{E}+04$ & $1.402 \mathrm{E}-07$ & -0.68 \\
\hline-1.1 & 153 & 5067 & $3.416 \mathrm{E}+00$ & $4.061 \mathrm{E}+04$ & $1.255 \mathrm{E}-07$ & -0.57 \\
\hline-1.2 & 167 & 4993 & $2.894 \mathrm{E}+00$ & $3.575 \mathrm{E}+04$ & $1.121 \mathrm{E}-07$ & -0.45 \\
\hline-1.3 & 182 & 4930 & $2.474 \mathrm{E}+00$ & $3.148 \mathrm{E}+04$ & $1.000 \mathrm{E}-07$ & -0.35 \\
\hline-1.4 & 197 & 4878 & $2.134 \mathrm{E}+00$ & $2.771 \mathrm{E}+04$ & $8.899 \mathrm{E}-08$ & -0.26 \\
\hline-1.5 & 211 & 4833 & $1.852 \mathrm{E}+00$ & $2.440 \mathrm{E}+04$ & $7.907 \mathrm{E}-08$ & -0.17 \\
\hline-1.6 & 225 & 4803 & $1.625 \mathrm{E}+00$ & $2.148 \mathrm{E}+04$ & $7.006 \mathrm{E}-08$ & -0.09 \\
\hline-1.7 & 239 & 4784 & $1.439 \mathrm{E}+00$ & $1.891 \mathrm{E}+04$ & $6.192 \mathrm{E}-08$ & -0.02 \\
\hline-1.8 & 254 & 4762 & $1.271 \mathrm{E}+00$ & $1.665 \mathrm{E}+04$ & $5.477 \mathrm{E}-08$ & 0.04 \\
\hline-1.9 & 268 & 4737 & $1.120 \mathrm{E}+00$ & $1.465 \mathrm{E}+04$ & $4.846 \mathrm{E}-08$ & 0.01 \\
\hline-2.0 & 282 & 4715 & $9.887 \mathrm{E}-01$ & $1.290 \mathrm{E}+04$ & $4.285 \mathrm{E}-08$ & 0.14 \\
\hline-2.1 & 296 & 4675 & $8.602 \mathrm{E}-01$ & $1.135 \mathrm{E}+04$ & $3.805 \mathrm{E}-08$ & 0.18 \\
\hline-2.2 & 310 & 4658 & $7.624 \mathrm{E}-01$ & $9.997 \mathrm{E}+03$ & $3.361 \mathrm{E}-08$ & 0.22 \\
\hline-2.3 & 323 & 4635 & $6.720 \mathrm{E}-01$ & $8.800 \mathrm{E}+03$ & $2.973 \mathrm{E}-08$ & 0.24 \\
\hline-2.4 & 337 & 4616 & $5.943 \mathrm{E}-01$ & $7.746 \mathrm{E}+03$ & $2.628 \mathrm{E}-08$ & 0.26 \\
\hline-2.5 & 351 & 4602 & $5.273 \mathrm{E}-01$ & $6.817 \mathrm{E}+03$ & $2.320 \mathrm{E}-08$ & 0.27 \\
\hline-2.6 & 364 & 4590 & $4.685 \mathrm{E}-01$ & $5.998 \mathrm{E}+03$ & $2.047 \mathrm{E}-08$ & 0.27 \\
\hline-2.7 & 378 & 4581 & $4.170 \mathrm{E}-01$ & $5.276 \mathrm{E}+03$ & $1.804 \mathrm{E}-08$ & 0.27 \\
\hline-2.8 & 392 & 4574 & $3.711 \mathrm{E}-01$ & $4.638 \mathrm{E}+03$ & $1.588 \mathrm{E}-08$ & 0.26 \\
\hline-2.9 & 406 & 4567 & $3.302 \mathrm{E}-01$ & $4.075 \mathrm{E}+03$ & $1.397 \mathrm{E}-08$ & 0.24 \\
\hline-3.0 & 419 & 4561 & $2.934 \mathrm{E}-01$ & $3.576 \mathrm{E}+03$ & $1.228 \mathrm{E}-08$ & 0.21 \\
\hline-3.1 & 433 & 4554 & $2.602 \mathrm{E}-01$ & $3.135 \mathrm{E}+03$ & $1.078 \mathrm{E}-08$ & 0.17 \\
\hline-3.2 & 447 & 4545 & $2.302 \mathrm{E}-01$ & $2.743 \mathrm{E}+03$ & $9.454 \mathrm{E}-09$ & 0.13 \\
\hline-3.3 & 462 & 4535 & $2.029 \mathrm{E}-01$ & $2.396 \mathrm{E}+03$ & $8.278 \mathrm{E}-09$ & 0.08 \\
\hline-3.4 & 476 & 4521 & $1.780 \mathrm{E}-01$ & $2.088 \mathrm{E}+03$ & $7.236 \mathrm{E}-09$ & 0.02 \\
\hline-3.5 & 491 & 4504 & $1.554 \mathrm{E}-01$ & $1.815 \mathrm{E}+03$ & $6.312 \mathrm{E}-09$ & -0.04 \\
\hline-3.6 & 506 & 4482 & $1.349 \mathrm{E}-01$ & $1.571 \mathrm{E}+03$ & $5.493 \mathrm{E}-09$ & -0.11 \\
\hline-3.7 & 521 & 4456 & $1.163 \mathrm{E}-01$ & $1.355 \mathrm{E}+03$ & $4.765 \mathrm{E}-09$ & -0.20 \\
\hline-3.8 & 537 & 4423 & $9.942 \mathrm{E}-02$ & $1.163 \mathrm{E}+03$ & $4.118 \mathrm{E}-09$ & -0.28 \\
\hline
\end{tabular}


Table A.2. Intergranular component derived from the inversion. Microturbulence is equal to $2.0 \mathrm{~km} \mathrm{~s}^{-1}$ and macroturbulence is $1.0 \mathrm{~km} \mathrm{~s}^{-1} . \tau_{5}$ stands for continuum optical depth at $5000 \AA, z$ for geometrical height, $T$ for temperature, $P_{\mathrm{e}}$ for electron pressure, $P_{\mathrm{g}}$ for gas pressure, $\rho$ for density, and $v_{\mathrm{LOS}}$ for line-of-sight velocity. Positive velocities indicate downflows. The gravitational redshift $\left(636 \mathrm{~m} \mathrm{~s}^{-1}\right)$ has been subtracted. The geometrical height scale is such that the model is in lateral pressure balance with the granular component.

\begin{tabular}{|c|c|c|c|c|c|c|}
\hline $\log \tau_{5}$ & $z(\mathrm{~km})$ & $T(\mathrm{~K})$ & $P_{\mathrm{e}}\left(\right.$ dyn $\left.\mathrm{cm}^{-2}\right)$ & $P_{\mathrm{g}}\left(\right.$ dyn $\left.\mathrm{cm}^{-2}\right)$ & $\rho\left(\mathrm{g} \mathrm{cm}^{-3}\right)$ & $v_{\mathrm{LOS}}\left(\mathrm{km} \mathrm{s}^{-1}\right)$ \\
\hline 1.0 & -82.9 & 8408 & $1.747 \mathrm{E}+03$ & $2.172 \mathrm{E}+05$ & $4.047 \mathrm{E}-07$ & 11.26 \\
\hline 0.9 & -76.9 & 8226 & $1.366 \mathrm{E}+03$ & $2.105 \mathrm{E}+05$ & $4.009 \mathrm{E}-07$ & 10.16 \\
\hline 0.8 & -70.9 & 8041 & $1.051 \mathrm{E}+03$ & $2.040 \mathrm{E}+05$ & $3.975 \mathrm{E}-07$ & 9.12 \\
\hline 0.7 & -65.0 & 7846 & $7.908 \mathrm{E}+02$ & $1.976 \mathrm{E}+05$ & $3.945 \mathrm{E}-07$ & 8.18 \\
\hline 0.6 & -59.0 & 7637 & $5.747 \mathrm{E}+02$ & $1.911 \mathrm{E}+05$ & $3.921 \mathrm{E}-07$ & 7.32 \\
\hline 0.5 & -52.7 & 7392 & $3.881 \mathrm{E}+02$ & $1.844 \mathrm{E}+05$ & $3.908 \mathrm{E}-07$ & 6.55 \\
\hline 0.4 & -45.9 & 7137 & $2.519 \mathrm{E}+02$ & $1.770 \mathrm{E}+05$ & $3.886 \mathrm{E}-07$ & 5.85 \\
\hline 0.3 & -38.2 & 6880 & $1.589 \mathrm{E}+02$ & $1.689 \mathrm{E}+05$ & $3.846 \mathrm{E}-07$ & 5.23 \\
\hline 0.2 & -29.5 & 6633 & $9.932 \mathrm{E}+01$ & $1.598 \mathrm{E}+05$ & $3.774 \mathrm{E}-07$ & 4.67 \\
\hline 0.1 & -19.4 & 6402 & $6.264 \mathrm{E}+01$ & $1.496 \mathrm{E}+05$ & $3.660 \mathrm{E}-07$ & 4.17 \\
\hline 0.0 & -8.0 & 6195 & $4.085 \mathrm{E}+01$ & $1.384 \mathrm{E}+05$ & $3.499 \mathrm{E}-07$ & 3.73 \\
\hline-0.1 & 4.7 & 6041 & $2.919 \mathrm{E}+01$ & $1.266 \mathrm{E}+05$ & $3.284 \mathrm{E}-07$ & 3.34 \\
\hline-0.2 & 18.2 & 5913 & $2.193 \mathrm{E}+01$ & $1.149 \mathrm{E}+05$ & $3.044 \mathrm{E}-07$ & 3.00 \\
\hline-0.3 & 32.3 & 5809 & $1.717 \mathrm{E}+01$ & $1.036 \mathrm{E}+05$ & $2.793 \mathrm{E}-07$ & 2.71 \\
\hline-0.4 & 46.9 & 5717 & $1.376 \mathrm{E}+01$ & $9.294 \mathrm{E}+04$ & $2.546 \mathrm{E}-07$ & 2.44 \\
\hline-0.5 & 61.7 & 5639 & $1.130 \mathrm{E}+01$ & $8.306 \mathrm{E}+04$ & $2.307 \mathrm{E}-07$ & 2.22 \\
\hline-0.6 & 76.8 & 5571 & $9.426 \mathrm{E}+00$ & $7.403 \mathrm{E}+04$ & $2.081 \mathrm{E}-07$ & 2.02 \\
\hline-0.7 & 91.9 & 5507 & $7.919 \mathrm{E}+00$ & $6.584 \mathrm{E}+04$ & $1.872 \mathrm{E}-07$ & 1.85 \\
\hline-0.8 & 107 & 5447 & $6.695 \mathrm{E}+00$ & $5.844 \mathrm{E}+04$ & $1.680 \mathrm{E}-07$ & 1.70 \\
\hline-0.9 & 122 & 5388 & $5.678 \mathrm{E}+00$ & $5.180 \mathrm{E}+04$ & $1.506 \mathrm{E}-07$ & 1.55 \\
\hline-1.0 & 137 & 5327 & $4.809 \mathrm{E}+00$ & $4.584 \mathrm{E}+04$ & $1.348 \mathrm{E}-07$ & 1.42 \\
\hline-1.1 & 152 & 5263 & $4.066 \mathrm{E}+00$ & $4.050 \mathrm{E}+04$ & $1.205 \mathrm{E}-07$ & 1.30 \\
\hline-1.2 & 168 & 5192 & $3.424 \mathrm{E}+00$ & $3.572 \mathrm{E}+04$ & $1.077 \mathrm{E}-07$ & 1.17 \\
\hline-1.3 & 183 & 5115 & $2.875 \mathrm{E}+00$ & $3.147 \mathrm{E}+04$ & $9.637 \mathrm{E}-08$ & 1.03 \\
\hline-1.4 & 198 & 5027 & $2.399 \mathrm{E}+00$ & $2.769 \mathrm{E}+04$ & $8.629 \mathrm{E}-08$ & 0.89 \\
\hline-1.5 & 213 & 4937 & $2.006 \mathrm{E}+00$ & $2.435 \mathrm{E}+04$ & $7.725 \mathrm{E}-08$ & 0.75 \\
\hline-1.6 & 227 & 4837 & $1.665 \mathrm{E}+00$ & $2.141 \mathrm{E}+04$ & $6.934 \mathrm{E}-08$ & 0.59 \\
\hline-1.7 & 242 & 4727 & $1.369 \mathrm{E}+00$ & $1.883 \mathrm{E}+04$ & $6.242 \mathrm{E}-08$ & 0.41 \\
\hline-1.8 & 256 & 4617 & $1.118 \mathrm{E}+00$ & $1.657 \mathrm{E}+04$ & $5.624 \mathrm{E}-08$ & 0.22 \\
\hline-1.9 & 269 & 4545 & $9.401 \mathrm{E}-01$ & $1.459 \mathrm{E}+04$ & $5.028 \mathrm{E}-08$ & 0.04 \\
\hline-2.0 & 283 & 4493 & $8.043 \mathrm{E}-01$ & $1.284 \mathrm{E}+04$ & $4.476 \mathrm{E}-08$ & -0.20 \\
\hline-2.1 & 296 & 4442 & $6.873 \mathrm{E}-01$ & $1.129 \mathrm{E}+04$ & $3.982 \mathrm{E}-08$ & -0.42 \\
\hline-2.2 & 309 & 4404 & $5.946 \mathrm{E}-01$ & $9.931 \mathrm{E}+03$ & $3.532 \mathrm{E}-08$ & -0.61 \\
\hline-2.3 & 323 & 4370 & $5.170 \mathrm{E}-01$ & $8.729 \mathrm{E}+03$ & $3.128 \mathrm{E}-08$ & -0.79 \\
\hline-2.4 & 336 & 4342 & $4.518 \mathrm{E}-01$ & $7.669 \mathrm{E}+03$ & $2.766 \mathrm{E}-08$ & -0.94 \\
\hline-2.5 & 349 & 4317 & $3.964 \mathrm{E}-01$ & $6.735 \mathrm{E}+03$ & $2.443 \mathrm{E}-08$ & -1.07 \\
\hline-2.6 & 362 & 4296 & $3.489 \mathrm{E}-01$ & $5.913 \mathrm{E}+03$ & $2.156 \mathrm{E}-08$ & -1.17 \\
\hline-2.7 & 375 & 4277 & $3.079 \mathrm{E}-01$ & $5.189 \mathrm{E}+03$ & $1.900 \mathrm{E}-08$ & -1.26 \\
\hline-2.8 & 388 & 4260 & $2.722 \mathrm{E}-01$ & $4.552 \mathrm{E}+03$ & $1.673 \mathrm{E}-08$ & -1.32 \\
\hline-2.9 & 401 & 4244 & $2.406 \mathrm{E}-01$ & $3.992 \mathrm{E}+03$ & $1.473 \mathrm{E}-08$ & -1.36 \\
\hline-3.0 & 414 & 4228 & $2.127 \mathrm{E}-01$ & $3.498 \mathrm{E}+03$ & $1.296 \mathrm{E}-08$ & -1.38 \\
\hline-3.1 & 427 & 4211 & $1.877 \mathrm{E}-01$ & $3.064 \mathrm{E}+03$ & $1.139 \mathrm{E}-08$ & -1.38 \\
\hline-3.2 & 440 & 4194 & $1.653 \mathrm{E}-01$ & $2.681 \mathrm{E}+03$ & $1.001 \mathrm{E}-08$ & -1.36 \\
\hline-3.3 & 453 & 4173 & $1.449 \mathrm{E}-01$ & $2.344 \mathrm{E}+03$ & $8.798 \mathrm{E}-09$ & -1.31 \\
\hline-3.4 & 466 & 4150 & $1.265 \mathrm{E}-01$ & $2.046 \mathrm{E}+03$ & $7.724 \mathrm{E}-09$ & -1.24 \\
\hline-3.5 & 479 & 4123 & $1.097 \mathrm{E}-01$ & $1.783 \mathrm{E}+03$ & $6.776 \mathrm{E}-09$ & -1.16 \\
\hline-3.6 & 493 & 4092 & $9.450 \mathrm{E}-02$ & $1.551 \mathrm{E}+03$ & $5.938 \mathrm{E}-09$ & -1.05 \\
\hline-3.7 & 506 & 4056 & $8.060 \mathrm{E}-02$ & $1.345 \mathrm{E}+03$ & $5.197 \mathrm{E}-09$ & -0.92 \\
\hline-3.8 & 520 & 4014 & $6.796 \mathrm{E}-02$ & $1.163 \mathrm{E}+03$ & $4.538 \mathrm{E}-09$ & -0.77 \\
\hline
\end{tabular}




\section{References}

Allende Prieto, C., \& García López, R. 1997, A\&AS, 129, 41

Allende Prieto, C., Ruiz Cobo, B., \& García López, R. 1998, ApJ, 502, 951

Allende Prieto, C., Barklem, P. S., Asplund, M., \& Ruiz Cobo, B. 2001, ApJ, 558, 830

Anstee, S. D., \& O'Mara, B. J. 1995, MNRAS, 276, 859

Asplund, M., Nordlund, A., Trampedach, R., Allende Prieto, C., Stein, R. F. 2000a, A\&A, 359, 729

Asplund, M., Nordlund, A., Trampedach, R., \& Stein, R. F. 2000b, A\&A, 359, 743

Athay, R. G., \& Lites, B. W. 1972, ApJ, 176, 809

Bard, A., Kock, A., \& Kock, M. 1991, A\&A, 248, 315

Bard, A., \& Kock, M. 1994, A\&A, 282, 101

Barklem, P. S., \& O'Mara, B. J. 1997, MNRAS, 290, 102

Barklem, P. S., Anstee, S. D., \& O'Mara, B. J. 1998, PASA, 15, 336

Barklem, P. S., Piskunov, N., \& O'Mara, B. J. 2000, A\&AS, 142,467

Bellot Rubio, L. R., Ruiz Cobo, B., \& Collados, M. 1998, ApJ, 506,805

Bellot Rubio, L. R., Ruiz Cobo, B., \& Collados, M. 1999, A\&A, 341, L31

Blackwell, D. E., Petford, A. D., \& Simmons, G. J. 1982, MNRAS, 201, 595

Blackwell, D. E., Booth, A. J., Haddock, D. J., Petford, A. D., \& Legget, S. K. 1986, MNRAS, 220, 549

Blackwell, D. E., Lynas-Gray, A. E., Smith, G. 1995a, A\&A, 296,217

Blackwell, D. E., Smith, G., \& Lynas-Gray, A. E. 1995b, A\&A, 303,575

Brault, J., \& Neckel, H. 1987, Spectral Atlas of Solar Absolute Disk-Averaged and Disk-Center Intensity from 3290 to $12510 \AA$ A, unpublished

Bruls, J. H. M. J., Rutten, R. J., \& Shchukina, N. G. 1992, A\&A, 265, 237

Frutiger, C., Solanki, S. K., Fligge, M., \& Bruls, J. H. 2000, A\&A, 358, 1109
Gingerich, O., Noyes, R. W., \& Kalkofen, W. 1971, Sol. Phys., 18,347

Hannaford, P., Lowe, R. M., Grevesse, N., \& Noels, A. 1992, A\&A, 259, 310

Holweger, H., \& Müller, E. A. 1974, Sol. Phys., 39, 19

Holweger, H., Heise, C., \& Kock, M. 1990, A\&A, 232, 510

Holweger, H., Bard, A., Kock, A., \& Kock, M. 1991, A\&A, 249, 545

Holweger, H., Kock, M., \& Bard, A. 1995, A\&A, 296, 233

Kroll, S., \& Kock, M. 1987, A\&AS, 67, 255

Kurucz, R. L., Furenlid, I., \& Brault, J. 1984, NSO Atlas, Sunspot, New Mexico

May, M., Ritcher, J., \& Whichelmann, J. 1974, A\&AS, 18, 405

Mihalas, D. 1967, in Methods in Computational Physics, vol. 7, ed. B. Alder (Academic Press, New York)

Nave, G., Johansson, S., Learner, R. C. M., Thorne, A. P., \& Brault, J. W. 1994, ApJS, 94, 221

Neckel, H. 1994, in The Sun as a Variable Star, IAU Coll., 143, 37

Neckel, H., \& Labs, D. 1994, Sol. Phys., 153, 91

Nesis, A., Hanslmeier, A., Hammer, R., et al. 1992, A\&A, 253, 261

O'Brian, T. R., Wickliffe, M. E., Lawler, J. E., Whaling, I. W., \& Brault, W. 1991, JOSA, 8,1185

O'Mara, B. J. 1976, MNRAS, 177, 551

Schnabel, R., Kock, M., \& Holweger, H. 1999, A\&A, 342, 610

Shchukina, N., \& Trujillo Bueno, J. 2001, ApJ, 550, 970

Ruiz Cobo, B., \& Del Toro Iniesta, J. C. 1992, ApJ, 398, 375

Ruiz Cobo, B., del Toro Iniesta, J. C., Rodríguez Hidalgo, I., Collados, M., \& Sánchez Almeida, J. 1996, Cool Stars, Stellar Systems and the Sun (9th Cambridge Workshop) ASP Conf. Ser., 109, 155

Ruiz Cobo, B. 1998, Ap\&SS, 263, 331

Thévenin, F. 1989, A\&AS, 77, 137

Thévenin, F. 1990, A\&AS, 82, 17

Wittmann, A. 1974, Sol. Phys., 35, 11 